\title{
ON THE REPRESENTATIONS OF A NUMBER AS THE SUM OF THREE SQUARES
}

\author{
BY \\ PAUL T. BATEMAN( $\left.{ }^{1}\right)$
}

1. Introduction. Let $r_{s}(n)$ denote the number of representations of the positive integer $n$ as the sum of $s$ squares, that is, the number-theoretic function defined by

$$
1+\sum_{n=1}^{\infty} r_{8}(n) e^{\pi i \tau n}=\left(\sum_{n=-\infty}^{\infty} e^{\pi i \tau n^{2}}\right)^{s}=\vartheta_{3}(0 \mid \tau)^{s}
$$

where $\tau$ is a variable in the half-plane $\Im(\tau)>0$. For $s=5,6,7,8$, Hardy $[1,2,3]\left({ }^{2}\right)$ proved that $r_{s}(n)$ is exactly equal to

$$
\rho_{s}(n)=\frac{\pi^{s / 2}}{\Gamma(s / 2)} n^{s / 2-1} \mathfrak{S}_{s}(n) ;
$$

here $\mathfrak{S}_{8}(n)$ is the so-called singular $\operatorname{series}\left({ }^{3}\right)$

$$
\mathfrak{S}_{8}(n)=\sum_{k=1}^{\infty} B_{k}(n), \quad B_{k}(n)=k^{-s / 2} \sum_{h \bmod 2 k} \eta(h, k)^{s} e^{-\pi i h n / k},
$$

where $\eta(h, k)=0$ if $(h, k)>1$ and

$$
\eta(h, k)=\frac{1}{2} k^{-1 / 2} \sum_{j \bmod 2 k} e^{\pi i h j^{2} / k}=\frac{1}{2}\left\{1+(-1)^{h k}\right\} k^{-1 / 2} \sum_{j=1}^{k} e^{\pi i h j^{2} / k}
$$

if $(h, k)=1$. He remarked: "When $s=2$ or $s>8$, this conclusion is false. The cases $s=3$ and $s=4$ are exceptional. The conclusion is true, but new difficulties arise in the proof because the series used are not all absolutely convergent. These difficulties are easily surmounted when $s=4$, but are more serious when $s=3$."

It is the purpose of this paper to show that in spite of these convergence

Presented to the Society, August 22, 1946; received by the editors January 15, 1947 and, in revised form, September 28, 1950.

(1) The author wishes to acknowledge his indebtedness to Professor Hans Rademacher, who suggested this problem and gave much helpful advice.

(2) Numbers in brackets refer to the bibliography at the end of the paper. The Hardy proof is summarized in Hardy [1,2], given in detail for $s=5$ and $s=8$ in Hardy [3], given in detail for $s=7$ in Stanley [1], given in a revised form by A. Oppenheim and G. Pall in Dickson [2, chap. 13], and given for $s=8$ in Hardy [5, pp. 148-153]. There is also an elegant treatment in Estermann [2]. We shall discuss the method of proof in more detail in \$2.

(3) Logically, $B_{k}(n)$ should have a subscript or superscript to indicate its dependence on $s$. 
difficulties, the Hardy proof can essentially be carried over to these cases. The case $s=4$ is rather easy, for we shall see ( $\$ 3$ ) that absolute convergence can be restored by the device of grouping together certain terms $\left({ }^{4}\right)$. For $s=3$, more powerful methods are needed; however we shall show $(\$ \$ 4,5,6,7)$ that this case can be successfully handled by supplementing the formalities of the Hardy method with a limit process of the type used by Hecke $[1 ; 2]$. Naturally the results obtained in both cases agree with the classical results obtained from the study of quadratic forms (cf. Bachmann [2, chap. 6] and Dickson [1, chaps. 7 and 8$]$ ).

In the case $s=2$, the Hardy proof is no longer even formally correct. Actually $\rho_{2}(n)=2 r_{2}(n)$, as was asserted by Ramanujan $[1, \S 15]$ and as is easily proved by summing the singular series and comparing the result with the known formula for $r_{2}(n)$.

For $s>4$, the singular series $\Im_{s}(n)$ is positive and $\left|\Im_{s}(n)-1\right|$ has an upper bound less than unity; also the series

$$
\sum_{k=1}^{\infty} \sum_{h \bmod 2 k}\left|k^{-8 / 2} \eta(h, k)^{s} e^{-\pi i h n / k}\right|
$$

converges uniformly in $n$ (in fact its value is $\{\zeta(s / 2-1)\} /\left\{\left(1-2^{-s / 2}\right) \zeta(s / 2)\right\}$, where $\zeta$ denotes the Riemann zeta function). For $s=4$, the singular series converges to a positive value, but this value $\mathfrak{S}_{4}(n)$ is unbounded above and also can be arbitrarily close to zero (cf. the remark at the end of $\$ 3$ ); moreover the series (1.04) diverges, although the series

$$
\sum_{k=1}^{\infty}\left|\sum_{h \bmod 2 k} k^{-s / 2} \eta(h, k)^{s} e^{-\pi i h n / k}\right|
$$

does converge-but not, of course, uniformly in $n$ (cf. the beginning of $\S 3$ ). For $s=3,2$, the singular series converges to a non-negative value, but this value may be zero and on the other hand is unbounded above (cf. Hardy and Wright $[1, \S 18.7]$ and the remarks at the end of $\$ 4$ of this paper); furthermore the singular series is not absolutely convergent, even in the sense of (1.05). The convergence difficulties that arise in the Hardy proof itself in the cases $s=3,4$ are even more serious, as we shall point out in $\S \S 3$ and 4 .

The idea of using modular functions to find exact formulas for $r_{s}(n)$ stems from Mordell. Shortly before Hardy's work he [1] successfully treated the case of even $s$ without, however, starting from the suggestive singular series. He in fact showed that the method could be extended to give the other arithmetical functions which must be added to $\rho_{s}(n)$ to give the exact formulas for even $s$ greater than 8. After Hardy's work he [2] was able to

(4) This remark essentially goes back to Mordell [1, p. 97]. Kloostermann [1] has applied similar methods to get exact formulas for the number of representations of a number in the form $x^{2}+y^{2}+c z^{2}+c t^{2}$ for $c=3,5,6,7$. 
extend this to odd $s$ greater than 8 . The real reason why $\rho_{s}(n)$ alone does not give exact formulas for $s>8$ was explained by Siegel [1, pp. 581-582].

The singular series is, for any $s$, just the Fourier series of $\pi^{-s / 2} \Gamma(s / 2) n^{1-s / 2} r_{s}(n)$, in the sense in which that term is used in the theory of almost periodic arithmetical functions (cf. Kac [1]). It arises naturally also if we seek merely to find an asymptotic formula for $r_{s}(n)$ by the circle method of Hardy, Littlewood, and Ramanujan. In fact Hardy and Ramanujan proved by this method (cf. Hardy [1] and Ramanujan [1, §15])

$$
r_{s}(n)=\rho_{s}(n)+O\left(n^{s / 4}\right)
$$

Naturally Hardy and Ramanujan did not publish this particular application of the circle method in detail, inasmuch as exact formulas are available in this case $\left({ }^{5}\right)$.

The singular series $\mathfrak{S}_{s}(n)$ can be summed in a number of ways. The most general method is due to Hardy $[3 ; 4]$ and rests on the fact that $B_{k}(n)$ is multiplicative in $k$; this method is useful for all $s \geqq 2$ and is in fact the method which we shall use ( $\$ 4)$ for summing the series in the case $s=3$. Ramanujan [1, §\$10-11 and 14-15] sketched two methods which work for all even $s$; he used them to prove that $\rho_{s}(n)$ gives the known formulas for $r_{s}(n)$ for $s=4,6,8$. Estermann [1, pp. 194-195] gave a method which can be applied for all even $s$, but which appears in the literature only for $s$ divisible by 8 (cf. Hardy [5, pp. 139-146]); it is the method which is easiest to use for $s=2$ to verify that $\rho_{2}(n)=2 r_{2}(n)$. Finally we show in $\S 3$ how for $s=4$ an evaluation of the singular series comes out as a by-product of the Hardy method; the same is true for all even $s \geqq 4$.

2. Notation, general lemmas, and method. Throughout the paper the letter $p$ will denote the general odd prime, $C_{1}, C_{2}, \cdots$ will stand for positive absolute constants, and $C_{1}(\tau), C_{2}(\tau), \ldots$ will denote positive constants depending only upon the parameter $\tau$. As usual $(n / k)$ will denote the symbol of quadratic residuacity; we shall understand it to be zero if $(n, k)>1$, unity if $k=1$, and defined in the customary way otherwise (cf. Landau [4, Definitions 18-19]). Further $\sum_{j \bmod k}^{\prime}$ will mean that $j$ runs through a reduced residue system modulo $k$. Other notations will be explained as used.

We must make an agreement about powers of the form $(h i-k i \tau)^{1 / 2}$ and $(h-k \tau)^{1 / 2}$, where $h$ and $k$ are integers and $\tau$ is a complex variable in the half-plane $J(\tau)>0$. For $k \neq 0$, the quantity in parentheses varies in a certain half-plane and thus there is no difficulty in defining the square root. In all cases (even for $k=0$ ) we agree to take that branch which has values with

(5) Professor Rademacher of ten proves (1.06) in his lectures, inasmuch as it is the simplest application of the circle method. Kloostermann (cf. [2, p. 407]) has proved a more general estimate of this type for the number of representations of a positive integer $n$ in the form $a_{1} x_{1}^{2}+\cdots+a_{8} x_{s}^{2}$, where $a_{1}, \cdots, a_{s}$ are positive integers and $s \geqq 5$. Kloostermann's result would give (1.06) as a special case, but with an exponent $s / 4+\epsilon$ in the error term. 
positive real part for purely imaginary $\tau$. The case $h=0, k<0$ will not occur, and consequently our definition is unambiguous. Our choice of branch coincides with the usual principal value of the square root except for the case of $(h i-k i \tau)^{1 / 2}$ with $k<0$, for which we take the square root in the upper or lower half-plane according as $h$ is positive or negative. Powers of the form $(h i-k i \tau)^{s / 2}$ and $(h-k \tau)^{s / 2}$ will be understood to mean the ordinary sth powers of the square roots specified above. (Throughout, $s$ is a positive integer.) All other powers will have their usual principal values.

It is well known that $\eta(h, k)$ is an eighth root of unity if $h$ and $k$ are relatively prime and of opposite parity; otherwise $\eta(h, k)$ is zero. In fact (for $h$ any integer and $k$ a positive integer) we have

$$
\eta(h, k)= \begin{cases}\left(\frac{h}{k}\right) e^{-\pi i(k-1) / 4} & \text { for } h \text { even, } k \text { odd } \\ \left(\frac{k}{|h|}\right) e^{\pi i h / 4} & \text { for } h \text { odd, } k \text { even. }\end{cases}
$$

Also, for any positive integers $h$ and $k$, we have the reciprocity formula $\eta(h, k)=e^{\pi i / 4} \eta(-k, h)$. (For these facts see, for example, Landsberg [1, $\left.\$ 2\right]$ or Bachmann [1, chap. 7].)

For our purposes it is useful to extend (artificially) the definition of $\eta(h, k)$ to negative values of $k$, provided $h \neq 0$, through the equation

$$
\eta(h, k)=\eta(-h,-k) e^{(\operatorname{sign} h) \pi i / 2} \quad(h \neq 0, k \neq 0) .
$$

With this convention we have the more general reciprocity formula

$$
\eta(h, k)=\left\{\begin{array}{lr}
e^{\pi i / 4} \eta(-k, h) & \text { if } h>0, k \neq 0, \text { or if } h \neq 0, k>0, \\
e^{-3 \pi i / 4} \eta(-k, h) & \text { if } h<0, k<0 .
\end{array}\right.
$$

Also it is easy to check that in view of (2.02) and our convention about square roots we have

$$
\frac{\eta(h, k)}{(h i-k i \tau)^{1 / 2}}=\frac{\eta(-h,-k)}{\{(-h) i-(-k) i \tau\}^{1 / 2}} \quad(h \neq 0, k \neq 0) .
$$

Further, the reciprocity law (2.03) gives

$$
\frac{\eta(h, k)}{(h i-k i \tau)^{1 / 2}}=\frac{\eta(-k, h)}{(h-k \tau)^{1 / 2}} \quad(h \neq 0, k \neq 0) .
$$

The following lemmas will also be essential.

LEMMA 2.1. If $k$ is an even positive integer and $h$ is an odd integer, then

$$
(-1)^{(h+1) k / 4}\left(\frac{k}{|h+k|}\right)=\left(\frac{k}{|h|}\right) \text {. }
$$


This is easy to deduce from the quadratic reciprocity law and its second complement (Landau [4, Satz 93-95]).

Lemma 2.2. If $k$ is a positive integer and $h$ is an odd integer, then

$$
\eta(h+k, k)=\left(\frac{k}{|h|}\right) e^{-\boldsymbol{x} i h(k-1) / 4} .
$$

In view of the formulas (2.01) this result follows from the quadratic reciprocity law if $k$ is odd and from Lemma 2.1 if $k$ is even.

LEMMA 2.3. If $h$ is an odd integer and $k$ is any integer, the expression

$$
\left(\frac{k}{|h|}\right) \frac{e^{-\pi i h(k-1) / 4}}{(h i-k i \tau)^{1 / 2}}
$$

is unchanged if we replace $h$ by $-h$ and $k$ by $-k$.

This is easy to check if we recall the convention made earlier concerning $(h i-k i \tau)^{1 / 2}$ and use the first complement of the quadratic reciprocity law (Landau [4, Satz 92]).

Lemma 2.4. If $X$ is a nonprincipal residue character modulo $k$, if $m$ is an integer relatively prime to $k$, and if $g$ is any integer, then

$$
\left|\sum_{a \leqq \nu \leqq b} X(g+m \nu)\right|<2 k^{1 / 2} \log k .
$$

This is a generalization of a theorem of Pólya [1] and can be proved by combining the arguments of Schur [1, p. 34] and Landau [3, pp. 85-86].

In terms of our notation (which is a little less cumbersome than Hardy's) the proof given by Hardy of the equality $r_{s}(n)=\rho_{s}(n)$ for $5 \leqq s \leqq 8$ runs roughly as follows $\left({ }^{6}\right)$. For $J(\tau)>0$ he compares the two functions

$$
\begin{aligned}
\Psi_{s}(\tau) & =1+\sum_{n=1}^{\infty} \rho_{s}(n) e^{\pi i \tau n} \\
& =1+\sum_{n=1}^{\infty} e^{\pi i \tau n} \frac{\pi^{s / 2}}{\Gamma(s / 2)} n^{s / 2-1} \sum_{k=1}^{\infty} k^{-s / 2} \sum_{h \bmod 2 k} \eta(h, k)^{s} e^{-\pi i h n / k}
\end{aligned}
$$

and

$$
\vartheta_{3}(0 \mid \tau)^{s}=1+\sum_{n=1}^{\infty} r_{s}(n) e^{\pi i \tau n}=\left(\sum_{n=-\infty}^{\infty} e^{\pi i \tau n^{2}}\right)^{s},
$$

with a view toward showing that they are identical. The finite inner sum in

(6) We introduce this brief sketch of Hardy's proof in order that we may discuss where his argument breaks down for $s=3,4$. Also, it enables us to abbreviate the presentation of our proofs for $s=3,4$. 
the above definition (2.06) of $\Psi_{s}(\tau)$ is less in absolute value than $k$ and thus by absolute convergence the two infinite summations may be permuted (for $s \geqq 5$ ); this gives

$$
\Psi_{s}(\tau)=1+\sum_{k=1}^{\infty} \sum_{h \bmod 2 k} k^{-s / 2} \eta(h, k)^{s} \frac{\pi^{s / 2}}{\Gamma(s / 2)} \sum_{n=1}^{\infty} n^{s / 2-1} e^{\pi i(\tau-h / k) n}
$$

The following relation of Lipschitz (cf. Lipschitz [1, §§1-2] or Dickson [2, pp. 206-207])

$$
\frac{\pi^{\lambda}}{\Gamma(\lambda)} \sum_{n=1}^{\infty} n^{\lambda-1} e^{\pi i \tau n}=\sum_{m=-\infty}^{\infty} \frac{1}{(2 m i-i \tau)^{\lambda}} \quad(\lambda>1, \Im(\tau)>0)
$$

and formula (2.04) then lead to

$$
\begin{aligned}
\Psi_{s}(\tau) & =1+\sum_{k=1}^{\infty} \sum_{h=-\infty}^{\infty} \frac{\eta(h, k)^{s}}{(h i-k i \tau)^{s / 2}} \\
& =1+\frac{1}{(-i \tau)^{s / 2}}+\frac{1}{2} \sum_{k \neq 0} \sum_{h \neq 0} \frac{\eta(h, k)^{s}}{(h i-k i \tau)^{s / 2}} .
\end{aligned}
$$

(In the actual application of Hardy's method in this paper, specifically in $\S \S 3,6,7$, we shall use the notation $h \neq 0$ or $k \neq 0$ under a summation sign to indicate that $h$ or $k$, respectively, is to run over all the integers other than zero; similarly for other restrictions on the variable of summation.) Since $s \geqq 5$, the double series of partial fractions in (2.09) is absolutely convergent (cf. Ford [1, pp. 151-152]). Using this fact in connection with (2.05), one easily finds from the second expression for $\Psi_{s}(\tau)$ in (2.09) that

$$
\Psi_{s}(-1 / \tau)=(-i \tau)^{s / 2} \Psi_{s}(\tau) .
$$

Also it is well known that $\vartheta_{3}(0 \mid \tau)^{8}$ satisfies the same functional equation (2.10) (cf. Landsberg $[1, \S 1]$ or Whittaker and Watson $[1$, p. 475]). Now in the theory of modular functions $\left({ }^{7}\right)$ it is proved that if a function $F(\tau)$ is analytic in the upper half-plane and satisfies the four conditions

$$
\begin{gathered}
F(\tau+2)=F(\tau), \quad F(-1 / \tau)=F(\tau), \\
\limsup _{\tau \rightarrow i \infty}|F(\tau)|<\infty, \quad \limsup _{\tau \rightarrow i \infty}|F(1-1 / \tau)|<\infty,
\end{gathered}
$$

(7) The modular group $\Gamma$ is the group of all substitutions of the form $\tau^{\prime}=(a \tau+b) /(c \tau+d)_{3}$ where $a, b, c, d$ are integers such that $a d-b c=1$. We are interested here in the subgroup $\Gamma$, (of index 3 in $\Gamma$ ) consisting of those substitutions such that either $a \equiv d \equiv 0(\bmod 2)$ or $b \equiv c$ $\equiv 0(\bmod 2)$. In the upper half-plane the region in which $|\tau|>1$ and $|R(\tau)|<1$, along with either the left-hand or the right-hand half of its boundary, constitutes a fundamental region for $\Gamma_{3}$. The fact quoted in the text is another way of saying that if a function is analytic in the upper half-plane, invariant under $\Gamma_{3}$, and bounded in the fundamental region of $\Gamma_{3}$, then it must be a constant (cf. Ford $[1$, p. 94]). For brief outlines of the theory of modular functions, see Dickson [2, pp. 202-204] or Hardy [5, pp. 146-148]. 
then it must be a constant. The ratio $\Phi_{s}(\tau)=\Psi_{s}(\tau) / \vartheta_{3}(0 \mid \tau)^{s}$ is analytic in the upper half-plane, since, in view of the relation

$$
\vartheta_{3}(0 \mid \tau)=\prod_{n=1}^{\infty}\left(1-e^{2 \pi i n \tau}\right)\left(1+e^{(2 n-1) \pi i \tau}\right)^{2}
$$

(cf. Whittaker and Watson $[1$, p. 473] or Hardy and Wright $[1, \S 19.8]$ ), the function $\vartheta_{3}(0 \mid \tau)$ has no zeros there. Also $\Phi_{8}(\tau)$ satisfies the first three of the conditions (2.11), the second of them by (2.10), the first and third because both $\Psi_{8}(\tau)$ and $\vartheta_{3}(0 \mid \tau)^{s}$ possess power series in $e^{\boldsymbol{\pi} i \tau}$ convergent in the upper half-plane and beginning with the constant term 1 . Hence, in order to prove that the functions $\Psi_{8}(\tau)$ and $\vartheta_{3}(0 \mid \tau)^{8}$ are identical (and thus $r_{8}(n)=\rho_{s}(n)$ for every positive integer $n)$, it suffices to show that $\lim \sup _{\tau \rightarrow i \infty}\left|\Phi_{s}(1-1 / \tau)\right|$ $<\infty$. This is effected as follows. Taking the first expression for $\Psi_{s}(\tau)$ in (2.09) and using Lemma 2.2, Lemma 2.3, and the absolute convergence of the double sum in (2.09), one easily obtains

$$
\begin{aligned}
\Psi_{s}\left(1-\frac{1}{\tau}\right) & =(-i \tau)^{s / 2} \sum_{h \text { odd }>0} \sum_{k}\left(\frac{k}{h}\right)^{s} \frac{e^{-\pi i(h k-h+1) s / 4}}{(-h i \tau-k i)^{s / 2}} \\
& =(-i \tau)^{s / 2} \sum_{h \text { odd }>0} e^{\pi i(h-1) s / 4} \sum_{k \bmod 8 h}\left(\frac{k}{h}\right)^{s} e^{-\pi i h k s / 4} \\
& \sum_{m=-\infty}^{\infty}\{-h i \tau-(k-8 h m) i\}^{-s / 2}
\end{aligned}
$$

By using (2.08) again (this time in the opposite direction) and the absolute convergence of the resulting iterated infinite sum, it is easy to obtain

$$
\Psi_{s}\left(1-\frac{1}{\tau}\right)=(-i \tau)^{s / 2} \sum_{m=1}^{\infty} e^{2 \pi i \tau m / 8} \frac{(2 \pi)^{s / 2}}{\Gamma(s / 2)}\left(\frac{1}{8} m\right)^{s / 2-1} \sum_{h \text { odd }} D_{h}(m),
$$

where

$$
\begin{aligned}
D_{h}(m) & =\frac{1}{8} h^{-s / 2} e^{\pi i(h-1) s / 4} \sum_{k \bmod 8 h}\left(\frac{k}{h}\right)^{s} e^{2 \pi i k\left(m-s h^{2}\right) /(8 h)} \\
& =\frac{1}{8} h^{-s / 2} e^{\pi i(h-1) s / 4} \sum_{j \bmod h g} \sum_{g \bmod 8}\left(\frac{h g-8 j}{h}\right)^{s} e^{2 \pi i(h o-8 j)\left(m-s h^{2}\right) /(8 h)}
\end{aligned}
$$

$$
= \begin{cases}0 & \text { if } m \neq \equiv s(\bmod 8), \\ h^{-s / 2} e^{-\pi i(h-1) s / 4} \sum_{j \bmod h}\left(\frac{2 j}{h}\right)^{s} e^{-2 \pi i j m / h}=B_{h}(m) & \text { if } m \equiv s(\bmod 8) .\end{cases}
$$


Since (cf. Landsberg $[1, \S 1]$ or Whittaker and Watson $[1$, p. 475])

$$
\vartheta_{3}(0 \mid 1-1 / \tau)=(-i \tau)^{1 / 2} e^{\pi i \tau / 4} \sum_{n=-\infty}^{\infty} e^{2 \pi i \tau n(n+1) / 2},
$$

it follows that

$$
\begin{aligned}
\Phi_{s}(1-1 / \tau) & =\frac{\Psi_{s}(1-1 / \tau)}{\vartheta_{3}(0 \mid 1-1 / \tau)^{s}} \\
& =\frac{\sum_{m>0, m \equiv s(\bmod 8)} e^{2 \pi i \tau m / 8} \frac{(2 \pi)^{s / 2}}{\Gamma(s / 2)}\left(\frac{1}{8} m\right)^{s / 2-1} \sum_{h \text { odd }>0} B_{h}(m)}{e^{\pi i \tau s / 4}\left(\sum_{n=-\infty}^{\infty} e^{2 \pi i \tau n(n+1) / 2}\right)^{s}} .
\end{aligned}
$$

So far all the arguments in Hardy's proof have been valid for any $s \geqq 5$, but at this point the condition $s \leqq 8$ comes into play. Under this condition the least positive integer congruent to $s$ modulo 8 is $s$ itself, so that $\Phi_{s}(1-1 / \tau)$ has a finite limit as $\tau \rightarrow i \infty$. Thus $\Phi_{s}(\tau)=\Psi_{s}(\tau) / \vartheta_{3}(0 \mid \tau)^{s}$ satisfies the fourth of the conditions (2.11) and hence must be identically 1 . This completes our version of Hardy's proof that $r_{s}(n)=\rho_{s}(n)$ for $5 \leqq s \leqq 8$. Of course (2.16) gives as a side result exact formulas for the number of representations of a non-negative integer as a sum of $s$ triangular numbers.

The difficulties involved in carrying over the above proof to the cases $s=4$ and $s=3$ will be discussed at the beginning of $\S \S 3$ and 4 respectively.

3 . The case $s=4$. In applying the Hardy method just sketched to the case $s=4$ we encounter a difficulty at the very beginning in interchanging the infinite summations in (2.06) in order to get (2.07). This is easily overcome as follows. Let us put (following Ramanujan [1]) $C_{k}(n)=\sum_{j \bmod k}^{\prime} e^{-2 \pi i j n / k}$. Then, in view of (2.01), $B_{k}(n)=k^{-2} \sum_{h \bmod 2 k} \eta(h, k)^{4} e^{-\pi i h n / k}$ has the value $k^{-2} C_{k}(n)$ if $k$ is odd and the value $-k^{-2} C_{2 k}(n)$ if $k$ is even. Using the multiplicativity of $C_{k}(n)$ in $k$ and its evaluations for $k$ a prime-power (cf. Hardy [4, pp. 264-265], Hardy [5, pp. 137-139]) we easily find that $\left|C_{k}(n)\right|$ $\leqq(k, n) \leqq n$. Thus $\left|B_{k}(n)\right| \leqq k^{-2} n$. This inequality easily allows us to make the desired interchange in order of summation, so that as in (2.09) we arrive at the following series of partial fractions

$$
\Psi_{4}(\tau)=\frac{1}{2} \sum_{k} \sum_{(h, k)=1, h \equiv k+1(\bmod 2)} \frac{(-1)^{k}}{(h-k \tau)^{2}} .
$$

We know that the series in (3.01) is convergent, because of the way in which it arose. Multiplying it by the absolutely convergent series $\sum_{k}$ odd >0 $k^{-2}$ and using the fact that the Dirichlet product of an absolutely convergent 
series and a convergent series is convergent (cf. Landau $[2, \S \S 184-185]$, we easily obtain

$$
\frac{\pi^{2}}{8} \Psi_{4}(\tau)=\frac{1}{2} \sum_{k} \sum_{h \equiv k+1(\bmod 2)} \frac{(-1)^{k}}{(h-k \tau)^{2}},
$$

where the condition $(h, k)=1$ has been removed. (This step is a departure from the method outlined in $\$ 2$, but is very convenient for even $s$.)

As it stands the iterated summation in (3.02) is convergent but not absolutely convergent. However, as remarked by Mordell [1], it becomes absolutely convergent if we group together the two terms with $h=2 \mu-1$, $k=2 \nu$ and $h=2 \mu, k=2 \nu-1$ respectively, where $\mu, \nu$ is any pair of integers. Thus we may reverse the order of summation in (3.02), and this fact enables us to show that

$$
\Psi_{4}(-1 / \tau)=-\tau^{2} \Psi_{4}(\tau) .
$$

A similar argument is needed in treating

$$
\frac{\pi^{2}}{8} \Psi_{4}\left(1-\frac{1}{\tau}\right)=\frac{1}{2} \tau^{2} \sum_{k} \sum_{h \text { odd }} \frac{(-1)^{k}}{(h \tau+k)^{2}} .
$$

Here we group together the two terms for which $h$ has a given value and $k$ has the values $2 m$ and $2 m+1$ respectively, $m$ being any integer. Reversing the order of summation in (3.04) and applying (2.08), we get

$$
\frac{\pi^{2}}{8} \Psi_{4}\left(1-\frac{1}{\tau}\right)=-2 \pi^{2} \tau^{2} e^{\pi i \tau} \sum_{n=0}^{\infty} \sigma(2 n+1) e^{2 \pi i \tau n},
$$

where $\sigma(m)$ denotes the sum of the divisors of $m$. Having arrived at (3.03) and (3.05), we easily conclude as in $\S 2$ that $\Psi_{4}(\tau)$ and $\vartheta_{3}(0 \mid \tau)^{4}$ are identically equal, so that $r_{4}(n)=\rho_{4}(n)$ for every positive integer $n$.

If we reverse the steps used to get (3.01), that is, if we apply the Lipschitz formula (2.08) in the reverse direction, we naturally come back to (2.07) and (2.06) (with $s=4$ ). However, if we apply this process to (3.02) we easily find

$$
\frac{\pi^{2}}{8} \Psi_{4}(\tau)=\frac{\pi^{2}}{8}+\pi^{2} \sum_{n=1}^{\infty} e^{\pi i r n} \sum_{d \mid n}(-1)^{(d-1)(n / d-1)} d,
$$

so that, in view of (2.06), $\rho_{4}(n)$ is 8 times the sum of the (odd) divisors of $n$ if $n$ is odd, and 24 times the sum of the odd divisors of $n$ if $n$ is even. Thus we have proved the following theorem.

TheOrem A. For any positive integer $n$ we have

$$
r_{4}(n)=\rho_{4}(n)=8 \sum_{d \mid n, 4 \nmid d} d .
$$


Since $\Psi_{4}(\tau)$ is identically equal to $\vartheta_{3}(0 \mid \tau)^{4},(3.05)$ and (2.15) show that if $n$ is a non-negative integer, the number of representations of $n$ as the sum of four triangular numbers is equal to $16 \sigma(2 n+1)$. This well known fact can of course be deduced from Theorem A itself, since the number of representations of $n$ as the sum of four triangular numbers is equal to the number of representations of $8 n+4$ as the sum of four odd squares, which in turn is equal to $r_{4}(8 n+4)-r_{4}(2 n+1)$.

In the case $s=4$ (and also for $s=6,8$ ) the main advantage of Hardy's method as compared with Mordell's earlier method is one of motivation. Hardy uses the singular series as in (2.06) to construct a function $\Psi_{s}(\tau)$ which is to be compared with $\vartheta_{3}(0 \mid \tau)^{s}$. On the other hand Mordell, in the case $s=4$, for example, merely defines $\Psi_{4}(\tau)$ by (3.02) and then proves it identical to $\vartheta_{3}(0 \mid \tau)^{4}$ as above. After that it is a simple matter to express $\Psi_{4}(\tau)$ as a power series in $e^{\pi i \tau}$ as in (3.06) above and equate the coefficient of $e^{\pi i \tau n}$ with $r_{4}(n)$. For odd $s$ the advantages of Hardy's method are more striking, for it would be difficult to find the appropriate series analogous to (3.02) without starting from the singular series; in fact Mordell found it impossible until after Hardy's work.

The above explicit evaluation of $\rho_{4}(n)$ shows that $\mathfrak{S}_{4}(n)=\rho_{4}(n) /\left(\pi^{2} n\right)$ can have order of magnitude anywhere from $1 / n$ to $\log \log n$. In fact if $n=2^{\lambda}$ $(\lambda=1,2, \cdots)$, then $\Im_{4}(n)=24 /\left(\pi^{2} n\right)$. On the other hand if $n$ is twice the $[\log k]$ th power of the product of the first $k$ primes, it is not hard to see that as $k \rightarrow \infty$ we have $\Im_{4}(n)=8 \sigma(n) /\left(\pi^{2} n\right) \sim 6 \pi^{-2} e^{\gamma} \log \log n$, where $\gamma$ is Euler's constant. (Cf. Landau [2, §59]. Actually $\lim \sup _{n \rightarrow \infty}\left\{\Im_{4}(n) / \log \log n\right\}$ $=6 \pi^{-2} e^{\gamma}$.)

4. The case $s=3$. Evaluation of the singular series. In applying the Hardy method sketched in $\$ 2$ to the case $s=3$, we cannot even make the original exchange of order of summation needed to go from (2.06) to (2.07). Further, the double series of partial fractions which we get formally, namely

$$
1+\frac{1}{(-i \tau)^{3 / 2}}+\frac{1}{2} \sum_{k \neq 0} \sum_{h \neq 0} \frac{\eta(h, k)^{3}}{(h i-k i \tau)^{3 / 2}},
$$

probably does not converge anyway. Thus we are obliged to modify the Hardy method somewhat. We arbitrarily start with the double series

$$
\begin{aligned}
\Psi_{3, \sigma}(\tau)=1 & +\sum_{k=1}^{\infty} \sum_{h=-\infty}^{\infty} \frac{\eta(h, k)^{3}}{(h i-k i \tau)^{3 / 2}|h i-k i \tau|^{\sigma}} \\
=1 & +\frac{1}{(-i \tau)^{3 / 2}|-i \tau|^{\sigma}} \\
& +\frac{1}{2} \sum_{k \neq 0} \sum_{h \neq 0} \frac{\eta(h, k)^{3}}{(h i-k i \tau)^{3 / 2}|h i-k i \tau|^{\sigma}},
\end{aligned}
$$


where $\sigma$ is a positive number and the two expressions for $\Psi_{3, \sigma}(\tau)$ are equivalent by (2.04). (It will be convenient later on to assume also that $\sigma \leqq 1 / 2$.) After proving in the present section that the singular series converges for $s=3$ and has a sum such that the definition $(2.06)$ of $\Psi_{3}(\tau)$ has meaning, we shall in $\S 5$ show that the iterated sum in (4.01) does converge for any positive $\sigma$ and that its sum $\Psi_{3, \sigma}(\tau)$ has the property $\lim _{\sigma \rightarrow 0} \Psi_{3, \sigma}(\tau)=\Psi_{3}(\tau)$. Thus the desired properties of $\Psi_{3}(\tau)$ can be inferred from a study of the properties of $\Psi_{3, \sigma}(\tau)$. In $\$ 6$ we shall study the effect of the substitution $\tau^{\prime}=-1 / \tau$ and in $\$ 7$ we shall study $\Psi_{3, \sigma}(1-1 / \tau)$ in order to determine the behavior of $\Psi_{3}(1-1 / \tau)$ as $\tau \rightarrow i \infty$.

After giving the above general outline of the procedure to be followed for $s=3$, we turn to the main task of this section, namely proving that the singular series $\Im_{3}(n)=\sum_{k=1}^{\infty} B_{k}(n)$ converges. Since clearly

$$
B_{k_{1} k_{2}}(n)=B_{k_{1}}(n) B_{k_{2}}(n)
$$$$
\text { if }\left(k_{1}, k_{2}\right)=1 \text {, }
$$

it is necessary first of all to evaluate $B_{k}(n)$ for $k$ a prime-power. This has been done by Hardy [3, pp. 270-275], but in a notation slightly different from ours.

In [3] Hardy used the singular series in the slightly less convenient form

$$
\mathfrak{S}_{8}(n)=\sum_{k=1}^{\infty} A_{k}(n), \quad A_{k}(n)=\sum_{h \bmod k}^{\prime}\left(k^{-1} \sum_{j \bmod k} e^{2 \pi i h j^{2} / k}\right)^{\bullet} e^{-2 \pi i h n / k}
$$

It is easily seen that

$$
A_{k}(n)= \begin{cases}B_{k}(n) & \text { if } 2 \nmid k, \\ 0 & \text { if } 2 \mid k, 4 \nmid k, \\ B_{k / 2}(n) & \text { if } 4 \mid k,\end{cases}
$$

so that the two forms of the singular series are equivalent, provided the even and odd numbered terms converge separately. This is certainly the case for $s \geqq 4$, since then $\sum_{k=1}^{\infty}\left|B_{k}(n)\right|$ converges, and also turns out to be the case for $s=3$ and $s=2$.

By referring to Hardy's formulas (cf. also Dickson [2, pp. 215-222]) and using (4.03) we find the following evaluations: if $\nu$ is an even positive integer,
(4.041) $B_{2^{\nu}}(n)=\left\{\begin{array}{l}0 \\ 2^{-\nu / 2} \cos 4^{-1}\left(2^{-\nu+2} n-3\right) \pi \\ 0\end{array}\right.$
if $2^{p-2} \nmid n$,
if $2^{\nu-2} \mid n, 2^{-v+2} n \equiv 3(\bmod 4)$,
if $2^{\nu-2} \mid n, 2^{-\nu+2} n \not \equiv 3(\bmod 4)$,
(4.042) $B_{p^{\nu}}(n)=\left\{\begin{array}{l}0 \\ -p^{-v / 2-1} \\ (p-1) p^{-p / 2-1}\end{array}\right.$
if $p^{\nu-1} \nmid n$,
if $p^{\nu-1} \mid n, p^{\nu} \nmid n$,
if $p^{p} \mid n$. 
If $\nu$ is an odd positive integer,
(4.043)

$$
B_{2^{\nu}}(n)= \begin{cases}0 & \text { if } 2^{\nu-1} \nmid n, \\ 2^{-\nu / 2} \cos 4^{-1}\left(2^{-v+2} n-3\right) \pi & \text { if } 2^{\nu-1} \mid n\end{cases}
$$
(4.044) $B_{p^{\nu}}(n)=\left\{\begin{array}{l}0 \\ p^{-(\nu+1) / 2}\left(\frac{-p^{-\nu+1} n}{p}\right) \\ 0\end{array}\right.$ if $p^{\nu-1} \nmid n$, if $p^{n-1} \mid n, p^{\natural} \nmid n$, if $p^{\nu} \mid n$.

For the remainder of this section the summation letters $k, l, m$ will run over the positive integers, with limitations as specified.

Lemma 4.1. If $n$ is a fixed positive integer, the series

$$
\sum_{k \text { squarefree }}\left(\frac{-4 n}{k}\right) \frac{1}{k}
$$

converges to the positive value

$$
K(-4 n) \sum_{(l, 4 n)=1} \frac{\mu(l)}{l^{2}},
$$

where $\mu(l)$ is the Möbius function and, in the notation of Landau [4, Part 4],

$$
K(-4 n)=\sum_{m}\left(\frac{-4 n}{m}\right) \frac{1}{m} .
$$

By Landau [4, Satz 99, 141, 152] the series for $K(-4 n)$ converges to a positive value. Also the series $\sum_{(l, 4 n)=1} \mu(l) l^{-2}$ is absolutely convergent and has a positive value, namely $1 /\left\{\sum_{(l, 4 n)=1} l^{-2}\right\}$. Hence Dirichlet multiplication of these two series yields a series which converges to the product of the respective sums (cf. Landau $[2, \S 185]$ ). But

$$
\begin{aligned}
\sum_{(l, 4 n)=1} \frac{\mu(l)}{l^{2}} \sum_{(m, 4 n)=1}\left(\frac{-4 n}{m}\right) \frac{1}{m} & =\sum_{(k, 4 n)=1}\left(\frac{-4 n}{k}\right) \frac{1}{k} \sum_{l^{2} m=k} \mu(l) \\
& =\sum_{k \text { squarefree, }(k, 4 n)=1}\left(\frac{-4 n}{k}\right) \frac{1}{k},
\end{aligned}
$$

so that Lemma 4.1 is proved.

If $p$ is an odd prime not dividing $n$, formulas (4.042) and (4.044) tell us that

$$
B_{p}(n)=\left(\frac{-n}{p}\right) \frac{1}{p}, \quad B_{p^{2}}(n)=B_{p^{3}}(n)=\cdots=0 .
$$

Thus for $k$ odd and prime to $n$ we have by (4.02) 


$$
B_{k}(n)=\left(\frac{-n}{k}\right) \frac{|\mu(k)|}{k}
$$

Hence by Lemma 4.1 the series

$$
\sum_{(k, 4 n)=1} B_{k}(n)=\sum_{k \text { odd }, k \text { squarefree }}\left(\frac{-n}{k}\right) \frac{1}{k}=\sum_{k \text { squarefree }}\left(\frac{-4 n}{k}\right) \frac{1}{k}
$$

converges to a positive value. (We introduce the factor 4 so that we may drop the condition $k$ odd and also so that $-4 n$ becomes a discriminant number, which facilitates reference to the literature.) Now by the formulas (4.04) the series

$$
\chi_{2}(n)=1+B_{2}(n)+B_{2^{2}}(n)+\cdots
$$

and

$$
\chi_{p}(n)=1+B_{p}(n)+B_{p^{2}}(n)+\cdots
$$

are finite in length. Since the series (4.05) is convergent and since the sum of a finite number of convergent series is convergent, we can by (4.02) assert the convergence of the singular series

$$
\begin{aligned}
\sum_{k=1}^{\infty} B_{k}(n)=\left\{1+B_{2}(n)\right. & \left.+B_{2^{2}}(n)+\cdots\right\} \\
& \cdot\left\{\prod_{p \mid n}\left(1+B_{p}(n)+B_{p^{2}}(n)+\cdots\right)\right\} \sum_{(k, 4 n)=1} B_{k}(n) .
\end{aligned}
$$

(Notice that the even and odd terms of the singular series converge separately, as stated above.) Furthermore, by (4.05) and Lemma 4.1 we have

$$
\sum_{k=1}^{\infty} B_{k}(n)=\chi_{2}(n)\left\{\prod_{p \mid n} \chi_{p}(n)\right\}\left\{\sum_{(l, 4 n)=1} \mu(l) l^{-2}\right\} K(-4 n) .
$$

Using (4.041) and (4.043) we evaluate $\chi_{2}(n)$. Suppose $a$ is that non-negative integer such that $4^{a} \mid n, 4^{a+1} \not n$. Then

$$
\begin{aligned}
\chi_{2}(n) & =1+B_{2}(n)+B_{2^{3}}(n)+\cdots+B_{2^{2 a-1}}(n)+B_{2^{2 a+1}}(n)+B_{2^{2 a+2}(n)} \\
& =1-1 / 2-1 / 2^{2}-\cdots-1 / 2^{a}+B_{2^{2 a+1}}(n)+B_{2^{2 a+2}}(n) .
\end{aligned}
$$

Putting $n^{*}=4^{-a} n$, we have the following results. If $n^{*} \equiv 7(\bmod 8), \chi_{2}(n)=0$; if $n^{*} \equiv 3(\bmod 8), \chi_{2}(n)=1 / 2^{a} ;$ if $n^{*} \equiv 1,2,5,6(\bmod 8), \chi_{2}(n)=3 / 2^{a+1}$.

Now we evaluate $\chi_{p}(n)$ for an odd prime $p$ dividing $n$. Suppose $b$ is that non-negative integer such that $p^{2 b} \mid n, p^{2 b+2}\left\{n\right.$. Then if $p^{2 b+1} \mid n, \chi_{p}(n)$ has $B_{p^{2 b+2}}(n)$ as its last term and thus is given by

$$
\chi_{p}(n)=1+(p-1) / p^{2}+\cdots+(p-1) / p^{b+1}-1 / p^{b+2},
$$


while if $p^{2 b+1} \nmid n, \chi_{p}(n)$ has $B_{p^{2 b+1}}(n)$ as its last term and thus is given by

$$
\chi_{p}(n)=1+\frac{p-1}{p^{2}}+\cdots+\frac{p-1}{p^{b+1}}+\left(\frac{-p^{-2 b} n}{p}\right) \frac{1}{p^{b+1}} .
$$

Thus if $p$ is an odd prime dividing $n$ we may write in either case

$$
\begin{aligned}
\chi_{p}(n)=\left(1-\frac{1}{p^{2}}\right)\left(1+\frac{1}{p}\right. & +\cdots \\
& \left.+\frac{1}{p^{b-1}}+\frac{1}{p^{b}}\left\{1-\left(\frac{-p^{-2 b} n}{p}\right) \frac{1}{p}\right\}^{-1}\right),
\end{aligned}
$$

where $b=b(p)$ is the largest integer such that $p^{2 b} \mid n$. In particular if $p \mid n$ but $p^{2} \nmid n$, then $\chi_{p}(n)=1-1 / p^{2}$. Note that in any case $\chi_{p}(n)$ is positive. Thus by (4.05) the series

$$
\sum_{k \text { odd }} B_{k}(n)=\left\{\prod_{p \mid n} \chi_{p}(n)\right\} \sum_{(k, 4 n)=1} B_{k}(n)
$$

has a positive value, while $\sum_{k=1}^{\infty} B_{k}(n)=\chi_{2}(n) \sum_{k \text { odd }} B_{k}(n)$ is positive unless $\chi_{2}(n)=0$, that is, unless $n$ is of the form $4^{a}(8 m+7)$.

By (1.01) and (4.06) we have

$$
\begin{aligned}
\rho_{3}(n) & =2 \pi n^{1 / 2} \Im_{3}(n)=2 \pi n^{1 / 2} \sum_{k=1}^{\infty} B_{k}(n) \\
& =2 \pi n^{1 / 2} \chi_{2}(n)\left\{\prod_{p \mid n} \frac{\chi_{p}(n)}{1-1 / p^{2}}\right\}\left\{\sum_{l \text { odd }} \frac{\mu(l)}{l^{2}}\right\} K(-4 n) .
\end{aligned}
$$

Using (4.07) we get

$$
\begin{aligned}
& \rho_{3}(n)=\frac{16}{\pi} n^{1 / 2} \chi_{2}(n) K(-4 n) \prod_{p^{2} \mid n}\left(1+\frac{1}{p}+\cdots\right. \\
&+\left.\frac{1}{p^{b-1}}+\frac{1}{p^{b}}\left\{1-\left(\frac{-p^{-2 b} n}{p}\right) \frac{1}{p}\right\}^{-1}\right) \\
&=\frac{16}{\pi} n^{1 / 2} \chi_{2}(n) K(-4 n) \prod_{p^{2} \mid n}\left(1+\frac{1}{p}\left\{1-\left(\frac{-p^{-2} n}{p}\right) \frac{1}{p}\right\}^{-1}\right. \\
&\left.+\cdots+\frac{1}{p^{b}}\left\{1-\left(\frac{-p^{-2 b} n}{p}\right) \frac{1}{p}\right\}^{-1}\right) .
\end{aligned}
$$

Now in view of the values of $\chi_{2}(n)$ it is clear that

$$
n^{1 / 2} \chi_{2}(n)=\left(4^{a} n^{*}\right)^{1 / 2} \chi_{2}\left(4^{a} n^{*}\right)=n^{* 1 / 2} \chi_{2}\left(n^{*}\right) ;
$$

also $K(-4 n)=K\left(-4 n^{*}\right)$; hence $\rho_{3}(n)=\rho_{3}\left(n^{*}\right)$. But since 


$$
K\left(-4 p^{2} g\right)=\left\{1-\left(\frac{-4 g}{p}\right) \frac{1}{p}\right\} K(-4 g)
$$

for any odd prime $p$ (and any positive integer $g$ ), we have

$$
\rho_{3}\left(n^{*}\right)=\sum_{d^{2} \mid n^{*}} P_{3}\left(n^{*} / d^{2}\right)
$$

where for $4 \nmid n$

$$
P_{3}(n)=\frac{16}{\pi} n^{1 / 2} \chi_{2}(n) K(-4 n), \quad \chi_{2}(n)= \begin{cases}0 & \text { if } n \equiv 7(\bmod 8) \\ 1 & \text { if } n \equiv 3(\bmod 8), \\ 3 / 2 & \text { if } n \equiv 1,2,5,6(\bmod 8) .\end{cases}
$$

Hence if we put more generally

$$
P_{3}(n)=\frac{G_{n}}{\pi} n^{1 / 2} K(-4 n), \quad G_{n}=\left\{\begin{aligned}
0 & \text { if } n \equiv 0,4,7(\bmod 8), \\
16 & \text { if } n \equiv 3(\bmod 8), \\
24 & \text { if } n \equiv 1,2,5,6(\bmod 8),
\end{aligned}\right.
$$

we have finally

$$
\rho_{3}(n)=\sum_{d^{2} \mid n} P_{3}\left(n / d^{2}\right) .
$$

The series $K(-4 n)$ (defined in Lemma 4.1) can be summed in finite form (cf. Landau [4, Satz 214, 217, 219]), but we shall not enter further into this part of the matter.

Chowla [1] has shown that there exists a positive constant $C_{1}$ such that for a certain infinite sequence of squarefree positive integers $q$ congruent to 1 modulo 4 we have $K(-4 q)>C_{1} \log \log 4 q$. This shows that for $n$ tending to infinity $P_{3}(n)=\Omega\left(n^{1 / 2} \log \log (4 n)\right)$, so that a fortiori $\rho_{3}(n)=\Omega\left(n^{1 / 2} \log \log (4 n)\right)$ and $\mathfrak{S}_{3}(n)=\Omega(\log \log (4 n))$. However Lemma 5.2 below shows that $\Im_{3}(n)=O(\log (4 n) \log \log (4 n))$ for all positive integers $n$. On the other side $\mathfrak{S}_{3}(n)$ is of course zero for one sixth of all positive integers $n$ (cf. Landau $[1$, pp. $305-306])$.

5. The case $s=3$. Proof of the limit property of $\Psi_{3, \sigma}(\tau)$. In $\$ 4$ we showed that for any positive integer $n$ the singular series $\mathfrak{S}_{3}(n)=\sum_{k=1}^{\infty} B_{k}(n)$ converges to a non-negative value given by (4.08). By Lemma 5.2 below we have

$$
\rho_{3}(n)=2 \pi n^{1 / 2} \Im_{3}(n)<C_{2} n^{1 / 2} \log (4 n) \log \log (4 n) .
$$

Thus the series $\sum_{n=1}^{\infty} \rho_{3}(n) e^{\pi i \tau n}$ converges for $J(\tau)>0$ and the function

$$
\Psi_{3}(\tau)=1+\sum_{n=1}^{\infty} \rho_{3}(n) e^{\pi i \tau n}
$$

is well defined. 
In this section we show that the double series in (4.01), the definition of $\Psi_{3, \sigma}(\tau)$, converges for $\sigma>0$ and that

$$
\lim _{\sigma \rightarrow 0} \Psi_{3, \sigma}(\tau)=\Psi_{3}(\tau) .
$$

We begin with a number of preliminary lemmas and remarks concerning the singular series.

We have spoken so far of the singular series $\sum_{k=1}^{\infty} B_{k}(n)$ only for $n$ a positive integer. However, the definition $B_{k}(n)=k^{-3 / 2} \sum_{h \bmod 2 k} \eta(h, k)^{3} e^{-\pi i h n / k}$ makes sense for any integer $n$ and in this section we shall in fact be obliged to deal with the case $n \leqq 0$. Fortunately the formulas (4.04) still hold good, for Hardy's derivation of them does not require that $n$ be positive.

All we really need to know about the singular series for $n \leqq 0$ is that the series $\sum_{\boldsymbol{k}=1}^{\infty} B_{k}(n) k^{-\sigma}$ converges for any positive $\sigma$ and is uniformly less than a constant times some power of $(|n|+1)$, say. However the singular series itself does actually converge for $n \leqq 0$. In fact for any $n$ the series $\sum_{k=1}^{\infty} B_{k}(n) k^{-\sigma}$ converges for $\sigma>-1 / 2$, although the convergence is absolute only for $\sigma>0$.

If $n$ is a negative integer such that $-n$ is not a square, then $(-4 n / m)$ is still a nonprincipal character modulo $|4 n|$; thus Lemma 4.1 , our demonstration in $\$ 4$ of the convergence of $\sum_{k=1}^{\infty} B_{k}(n)$, and formula (4.08) are all still valid. If $-n$ is a square, other considerations are necessary.

LEMMA 5.1. If $n$ is an integer such that $-n$ is not a square, then any partial sum of the (convergent) series

$$
\sum_{m=1}^{\infty}\left(\frac{-4 n}{m}\right) \frac{1}{m}
$$

is less in absolute value than $\log |4 n|$.

For the proof put $S_{k}=\sum_{m=1}^{k}(-4 n / m)$ and let $d$ be the largest value of $\left|S_{k}\right|$ for any positive integer $k$. (This maximum exists because, under the hypotheses, $(-4 n / m)$ is a nonprincipal character modulo $|4 n|$.) By partial summation we have

$$
\sum_{m=1}^{M}\left(\frac{-4 n}{m}\right) \frac{1}{m}=\sum_{m=1}^{M-1} \frac{S_{m}}{m(m+1)}+\frac{S_{M}}{M} .
$$

Thus if $M>d$,

$$
\begin{aligned}
\left|\sum_{m=1}^{M}\left(\frac{-4 n}{m}\right) \frac{1}{m}\right| & \leqq \sum_{m=1}^{d-1} \frac{m}{m(m+1)}+\sum_{m=d}^{M-1} \frac{d}{m(m+1)}+\frac{d}{M} \\
& =\sum_{m=1}^{d-1} \frac{1}{m+1}+1=\sum_{m=1}^{d} \frac{1}{m} .
\end{aligned}
$$


If $M \leqq d$ we clearly have

$$
\left|\sum_{m=1}^{M}\left(\frac{-4 n}{m}\right) \frac{1}{m}\right| \leqq \sum_{m=1}^{M} \frac{1}{m} \leqq \sum_{m=1}^{d} \frac{1}{m} .
$$

Since obviously $d \leqq|n|$, we have $\sum_{m=1}^{d} m^{-1}<1+\log d<\log |4 n|$, which proves Lemma 5.1.

LeMMA 5.2. If $-n$ is not a square, the series $\mathfrak{S}_{3}(n)=\sum_{k=1}^{\infty} B_{k}(n)$ converges and any partial sum thereof is less in absolute value than $C_{2} \log |4 n| \log \log |4 n|$.

The convergence was already pointed out above. From (4.06) we see that any partial sum of $\sum_{k=1}^{\infty} B_{k}(n)$ is less in absolute value than $2\left\{\prod_{p \mid n}(1-1 / p)^{-1}\right\} \pi^{2} / 8$ times the upper bound of the absolute values of the partial sums of $\sum_{m=1}^{\infty}(-4 n / m) m^{-1}$. But (cf. Hardy and Wright $[1, \S 22.8]$ )

$$
\frac{\pi^{2}}{4} \prod_{p \nmid n}\left(1-\frac{1}{p}\right)^{-1}=\frac{\pi^{2}}{4} \frac{|n|}{\phi(|n|)}<C_{2} \log \log |4 n|
$$

where $\phi$ denotes the Euler function. Thus Lemma 5.2 follows from Lemma 5.1 .

Lemma 5.3. If $n \neq 0$ and if $L$ is the largest square dividing $(n, k)$, then $\left|B_{k}(n)\right| \leqq 2 L^{1 / 2} / k \leqq 2(n, k)^{1 / 2} / k \leqq 2|n|^{1 / 2} / k$.

An examination of the formulas (4.04) reveals that (if $\nu$ is a positive integer)

$$
\left|B_{2^{\nu}}(n)\right| \leqq 2^{a+1} / 2^{\nu}, \quad\left|B_{p^{\nu}}(n)\right| \leqq p^{b} / p^{\nu}
$$

where $a$ is the largest integer such that $4^{a} \mid n$, and $b=b(p)$ is the largest integer such that $p^{2 b} \mid n, p$ being any odd prime. From this remark and the multiplicative property (4.02), the lemma follows.

Lemma 5.4. If $-n$ is not a square, then (i) $\sum_{k=1}^{\infty} B_{k}(n) k^{-\sigma}$ converges absolutely for $\sigma>0$, (ii) $\lim _{\sigma \rightarrow 0} \sum_{k=1}^{\infty} B_{k}(n) k^{-\sigma}=\sum_{k=1}^{\infty} B_{k}(n)$, (iii) the sum of the series $\sum_{k=1}^{\infty} B_{k}(n) k^{-\sigma}$ is less than $C_{2} \log |4 n| \log \log |4 n|$ for any positive $\sigma$.

Statement (i) follows from Lemma 5.3, (ii) follows from the convergence of $\sum_{k=1}^{\infty} B_{k}(n)$ and the standard continuity theorem for Dirichlet series (cf. Landau [2, pp. 106-107]), and (iii) is proved as follows. Put $U_{k}$ $=\sum_{m=1}^{k} B_{m}(n)$. Then by Lemma 5.2,

$$
\begin{aligned}
\sum_{k=1}^{\infty} B_{k}(n) k^{-\sigma} & =\sum_{k=1}^{\infty} U_{k}\left\{k^{-\sigma}-(k+1)^{-\sigma}\right\} \\
& <C_{2} \log |4 n| \log \log |4 n| \sum_{k=1}^{\infty}\left\{k^{-\sigma}-(k+1)^{-\sigma}\right\} \\
& =C_{2} \log |4 n| \log \log |4 n| .
\end{aligned}
$$


Thus Lemma 5.4 is proved.

Lемма 5.5. If $n<0$ and $-n$ is a square, then the series $\sum_{k=1}^{\infty} B_{k}(n) k^{-\sigma}$ converges absolutely for any positive $\sigma$ and its sum is uniformly less than 4 in absolute value.

Absolute convergence follows from Lemma 5.3. As before suppose $4^{a} \mid n$, $4^{a+1} \nmid n$, and for each odd prime $p$ dividing $n$, suppose $b=b(p)$ is such that $p^{2 b} \mid n, p^{2 b+2} \nmid n$. Under the hypotheses, $4^{-a} n \equiv 7(\bmod 8)$. Thus by $(4.02)$, the absolute convergence of $\sum_{k=1}^{\infty} B_{k}(n) k^{-\sigma}$, and the formulas (4.04), we have

$$
\begin{aligned}
\sum_{k=1}^{\infty} B_{k}(n) k^{-\sigma}= & \left\{1-\frac{1}{2^{1+\sigma}}-\frac{1}{2^{2+3 \sigma}}-\cdots-\frac{1}{2^{a+1+(2 a+1) \sigma}}-\frac{1}{2^{a+1+(2 a+2) \sigma}}\right\} \\
& \cdot \prod_{p \nmid n}\left\{1+\frac{1}{p^{1+\sigma}}\right\} \prod_{p \mid n}\left\{1+\frac{p-1}{p^{2+2 \sigma}}+\cdots\right. \\
& \left.\quad+\frac{p-1}{p^{b+1+2 b \sigma}}+\frac{1}{p^{b+1+(2 b+1) \sigma}}\right\} .
\end{aligned}
$$

The first factor here is zero for $\sigma=0$, and for $\sigma>0$ its derivative is easily seen to be less than $3 \log 2$; thus the first factor is less than $\min (1,3 \sigma \log 2)$. Also the product over the primes dividing $n$ is less than $\coprod_{p \mid n}\left\{1+p^{-1-2 \sigma}\right\}$. Hence

$$
\begin{aligned}
\sum_{k=1}^{\infty} B_{k}(n) k^{-\sigma} & <\min (1,3 \sigma \log 2) \prod_{p}\left(1+p^{-1-\sigma}\right) \\
& <\min (1,3 \sigma \log 2) \zeta(1+\sigma) \\
& <(1+1 / \sigma) \min (1,3 \sigma \log 2) \\
& \leqq 1+3 \log 2,
\end{aligned}
$$

which proves the lemma.

Lemma 5.6. If $\sigma>0$, the series $\sum_{k=1}^{\infty} B_{k}(0) k^{-\sigma}$ converges absolutely and has a sum less than unity.

For any positive integer $\lambda$, the formulas (4.04) give immediately

$$
\begin{aligned}
B_{2^{2 \lambda}}(0) & =0, & B_{2^{2 \lambda-1}}(0) & =-\frac{1}{2^{\lambda}}=-\frac{\phi\left(2^{\lambda}\right)}{2^{2 \lambda-1}}, \\
B_{p^{2 \lambda-1}}(0) & =0, & & B_{p^{2 \lambda}}(0)=\frac{p-1}{p^{\lambda+1}}=\frac{\phi\left(p^{\lambda}\right)}{p^{2 \lambda}} .
\end{aligned}
$$

Hence $B_{k}(0)=\phi_{1}\left(k^{1 / 2}\right) / k$ if $k$ is an odd square, $B_{k}(0)=-\phi\left(\{2 k\}^{1 / 2}\right) / k$ if $k$ is half an even square, and $B_{k}(0)=0$ otherwise. Thus $\sum_{k-1}^{\infty} B_{k}(0) k^{-\sigma}$ is absolutely convergent for $\sigma$ positive. Also 


$$
\begin{aligned}
\sum_{k=1}^{\infty} B_{k}(0) k^{-\sigma} & =\left\{1-\frac{1}{2^{1+\sigma}\left(1-2^{-1-2 \sigma}\right)}\right\} \prod_{p}\left\{1+\frac{p-1}{p^{2+2 \sigma}\left(1-p^{-1-2 \sigma}\right)}\right\} \\
& =\frac{1-2^{-1-\sigma}-2^{-1-2 \sigma}}{1-2^{-1-2 \sigma}} \prod_{p} \frac{1-p^{-2-2 \sigma}}{1-p^{-1-2 \sigma}} \\
& =\frac{\left(1+2^{-1-\sigma}\right)\left(1-2^{-\sigma}\right) \zeta(1+2 \sigma)}{\prod_{p}\left(1-p^{-2-2 \sigma}\right)^{-1}} .
\end{aligned}
$$

Hence

$$
\sum_{k=1}^{\infty} B_{k}(0) k^{-\sigma}<\frac{1+2^{-1-\sigma}}{1+2^{-\sigma}}\left(1-2^{-2 \sigma}\right) \zeta(1+2 \sigma)<1
$$

and thus Lemma 5.6 is proved.

Although we do not need it for later use, it seems of interest to point out that the singular series still converges in the cases discussed in the two preceding lemmas. Here the even and odd numbered terms do not converge separately. In fact the singular series converges precisely because the nonvanishing odd-numbered terms are positive and the nonvanishing even terms are negative, with enough mutual cancellation to produce convergence. By appealing to the basic continuity theorem for Dirichlet series it is easy to see what the sum of the singular series must be in each case if it is convergent. In fact, since

$$
\sum_{k=1}^{\infty} B_{k}(0) k^{-\sigma}=\frac{1}{\left(1+2^{-\sigma}\right)\left(1-2^{-1-\sigma}\right)} \frac{\left(1-2^{-2 \sigma}\right) \zeta(1+2 \sigma)}{\zeta(2+2 \sigma)}
$$

for positive $\sigma$, the series $\sum_{k=1}^{\infty} B_{k}(0)$, if convergent, must have the sum $2 \log 2 / \zeta(2)=12 \pi^{-2} \log 2$. If $n<0$ and $-n$ is a square, we have (for $\sigma>0$ )

$$
\begin{aligned}
\sum_{k=1}^{\infty} B_{k}(n) k^{-\sigma}= & \left\{1-\frac{1}{2^{a+1+(2 a+2) \sigma}}\right\}\left\{1-\frac{1}{2^{1+\sigma}\left(1-2^{-1-2 \sigma}\right)}\right\} \prod_{p}\left\{1+\frac{1}{p^{1+\sigma}}\right\} \\
& \cdot \prod_{p \mid n}\left\{( 1 + \frac { 1 } { p ^ { 1 + \sigma } } ) ^ { - 1 } \left(1+\frac{p-1}{p^{2+2 \sigma}}+\cdots\right.\right. \\
& \left.\left.\quad+\frac{p-1}{p^{b+1+2 b \sigma}}+\frac{1}{p^{b+1+(2 b+1) \sigma}}\right)\right\} \\
= & \frac{1-2^{-a-1-(2 a+2) \sigma}}{\left(1-2^{-1-2 \sigma}\right) \zeta(2+2 \sigma)}\left(1-2^{-\sigma}\right) \zeta(1+\sigma) \prod_{p \mid n}\{\cdots\}
\end{aligned}
$$

and so the series $\sum_{k=1}^{\infty} B_{k}(n)$, if convergent, must have the value $12 \pi^{-2}\left(1-2^{-a-1}\right) \log 2$.

In proving convergence in these two cases it is actually just as easy to 
prove that $\sum_{k=1}^{\infty} B_{k}(n) k^{-\sigma}$ converges for $\sigma>-1 / 2$. (We have convergence for $\sigma>-1 / 2$ also in case $-n$ is not a square, as is easily proved by a slight modification of the discussion in $\$ 4$.) Let us take the case $n=0$. The case of negative $n$ for which $-n$ is a square is similar, although more complicated in detail. Let $x$ be a positive integer greater than unity and put

$f_{0}(x)=\sum_{1 \leqq k \leqq x} \frac{\phi(k)}{k}, \quad f_{1}(x)=\sum_{1 \leqq k \leqq x, k \text { odd }} \frac{\phi(k)}{k}, \quad f_{2}(x)=\sum_{1 \leqq k \leqq x, k \text { even }} \frac{\phi(k)}{k}$.

Then

$$
\begin{aligned}
f_{0}(x) & =\sum_{k=1}^{x} \sum_{d \mid k} \frac{\mu(d)}{d}=\sum_{d=1}^{x} \frac{\mu(d)}{d}\left[\frac{x}{d}\right] \\
& =x \sum_{d=1}^{x} \frac{\mu(d)}{d^{2}}+O(\log x) \\
& =6 \pi^{-2} x+O(\log x) .
\end{aligned}
$$

Similarly

$$
f_{1}(x)=4 \pi^{-2} x+O(\log x), \quad f_{2}(x)=2 \pi^{-2} x+O(\log x) .
$$

Hence, using the evaluations of $B_{k}(0)$ given in the proof of Lemma 5.6, we have

$$
\sum_{k=1}^{x} B_{k}(0) k^{1 / 2}=f_{1}\left(x^{1 / 2}\right)-2^{1 / 2} f_{2}\left(\{2 x\}^{1 / 2}\right)=O(\log x),
$$

which shows that $\sum_{k=1}^{\infty} B_{k}(0) k^{-\sigma}$ converges for $\sigma>-1 / 2$ (cf. Landau [2, p. 121]).

LEMMA 5.7. If $\sigma \geqq 0$ and $\Im(\tau)=\beta>0$, then

$$
\sum_{m=-\infty}^{\infty} \frac{1}{(2 m i-i \tau)^{3 / 2}|2 m i-i \tau|^{\sigma}}=\sum_{n=-\infty}^{\infty} \frac{I(\sigma, n \beta)}{2 i \beta^{1 / 2+\sigma}} e^{\pi i \tau n}
$$

where

$$
I(\sigma, \rho)=\int_{1-i \infty}^{1+i \infty} \frac{e^{\pi \rho u}}{u^{(3+\sigma) / 2}(2-u)^{\sigma / 2}} d u .
$$

This lemma is a generalization of the Lipschitz formula (2.08) and is proved in the same way, namely, by applying the Poisson summation formula (cf. Mordell [3]).

Let us discuss the integral $I(\sigma, \rho)$ defined by (5.02). The integrand is a uniquely defined analytic function of $u$ in the $u$-plane with cuts along the negative real axis and along the positive real axis from +2 to $+\infty$. For $\rho \geqq 0$ 
we may deform the path of integration into a loop about the left-hand cut $\left(^{8}\right)$

$$
I(\sigma, \rho)=\int_{-\infty}^{\left(0^{+}\right)} \frac{e^{\pi \rho u}}{u^{(3+\sigma) / 2}(2-u)^{\sigma / 2}} d u \quad(\rho \geqq 0) .
$$

For $\rho \leqq 0$, we may deform the path of integration into a loop about the righthand cut

$$
I(\sigma, \rho)=\int_{\infty}^{\left(2^{-}\right)} \frac{e^{\pi \rho u}}{u^{(3+\sigma) / 2}(2-u)^{\sigma / 2}} d u \quad(\rho \leqq 0) ;
$$

if we restrict $\sigma$ to the interval $0 \leqq \sigma \leqq 1 / 2$, say, we have further

$$
I(\sigma, \rho)=2 i \sin \frac{\pi \sigma}{2} \int_{2}^{\infty} \frac{e^{\pi \rho u}}{u^{(3+\sigma) / 2}(u-2)^{\sigma / 2}} d u \quad(\rho \leqq 0) .
$$

These expressions are used in proving the following two lemmas.

Lemma 5.8. For $\sigma$ in the interval $0 \leqq \sigma \leqq 1 / 2$, the integral $I(\sigma, \rho)$ has the following estimates: If $\rho \geqq 0$, then $|I(\sigma, \rho)|<C_{3} e^{\pi_{\rho} / 2}$; if $\rho \leqq 0$, then $|I(\sigma, \rho)|$ $<C_{4} e^{2 \pi \rho}=C_{4} e^{-2 \pi|\rho|}$.

The first estimate follows from (5.03) by choosing the loop as follows: along the lower bank of the cut from $-\infty$ to $-1 / 2$, then counterclockwise on a circle of radius $1 / 2$ around the origin, and then along the upper bank of the cut from $-1 / 2$ to $-\infty$. The second estimate follows directly from (5.04).

LEMMA 5.9. If $\rho \geqq 0$, then

$$
\lim _{\sigma \rightarrow 0} I(\sigma, \rho)=I(0, \rho)=\frac{2 \pi i(\pi \rho)^{1 / 2}}{\Gamma(3 / 2)} .
$$

If $\rho \leqq 0$, then

$$
\lim _{\sigma \rightarrow 0} I(\sigma, \rho)=I(0, \rho)=0 .
$$

The first of these statements follows from (5.03) and the Hankel integral formula for the reciprocal of the gamma function (cf. Whittaker and Watson $[1$, p. 245]). The second follows from (5.04).

We now proceed with the main task of this section. First we must prove that the double series in (4.01) converges, so that we have a meaningful definition of $\Psi_{3, \sigma}(\tau)$. Let us take the double series in the first form given in (4.01),

$$
\Psi_{3, \sigma}(\tau)=1+\sum_{k=1}^{\infty} \sum_{h=-\infty}^{\infty} \frac{\eta(h, k)^{3}}{(h i-k i \tau)^{3 / 2}|h i-k i \tau|^{\sigma}} .
$$

$\left.{ }^{8}\right)$ We use the notation of Watson for loop integrals. The notation in (5.03) means that the path of integration starts at $-\infty$ on the lower bank of the cut along the negative real axis, encircles the origin in the positive direction, and returns to $-\infty$ on the upper bank of the cut. 
We consider the inner sum and transform it by Lemma 5.7:

$$
\begin{aligned}
\sum_{h=-\infty}^{\infty} \frac{\eta(h, k)^{3}}{(h i-k i \tau)^{3 / 2} \mid}|h i-k i \tau|^{\sigma} \\
=\sum_{h \bmod 2 k} \eta(h, k)^{3} k^{-3 / 2-\sigma} \sum_{m=-\infty}^{\infty}\{2 m i-i(\tau-h / k)\}^{-3 / 2} \\
\quad \cdot|2 m i-i(\tau-h / k)|^{-\sigma} \\
=\sum_{h \bmod 2 k} \eta(h, k)^{3} k^{-3 / 2-\sigma} \sum_{n=-\infty}^{\infty} \frac{I(\sigma, n \beta)}{2 i \beta^{1 / 2+\sigma}} e^{\pi i(\tau-h / k) n} \\
=\sum_{n=-\infty}^{\infty} \frac{I(\sigma, n \beta)}{2 i \beta^{1 / 2+\sigma}} B_{k}(n) k^{-\sigma} e^{\pi i \tau n},
\end{aligned}
$$

where of course $B_{k}(n)=k^{-3 / 2} \sum_{h \bmod 2 k} \eta(h, k)^{3} e^{-\pi i h n / k}$. By Lemmas 5.3 and 5.8 the series

$$
\sum_{n=-\infty}^{\infty} \frac{I(\sigma, n \beta)}{2 i \beta^{1 / 2+\sigma}} B_{k}(n) k^{-\sigma} e^{\pi i \tau n}
$$

is majorized by

$$
\begin{gathered}
C_{4} \beta^{-1 / 2-\sigma} \sum_{n=-\infty}^{-1}|n|^{1 / 2} k^{-1-\sigma} e^{-\pi|n \beta|}+\frac{1}{2} C_{4} \beta^{-1 / 2-\sigma}\left|B_{k}(0)\right| k^{-\sigma} \\
+C_{3} \beta^{-1 / 2-\sigma} \sum_{n=1}^{\infty} n^{1 / 2} k^{-1-\sigma} e^{-\pi n \beta / 2}
\end{gathered}
$$

Hence, since $\sum_{k=1}^{\infty}\left|B_{k}(0)\right| k^{-\sigma}$ converges (Lemma 5.6), the following double series is absolutely convergent:

$$
\sum_{k=1}^{\infty} \sum_{n=-\infty}^{\infty} \frac{I(\sigma, n \beta)}{2 i \beta^{1 / 2+\sigma}} B_{k}(n) k^{-\sigma} e^{\pi i \tau n}
$$

Thus by (5.05) the double series

$$
\sum_{k=1}^{\infty} \sum_{h=-\infty}^{\infty} \frac{\eta(h, k)^{3}}{(h i-k i \tau)^{3 / 2}|h i-k i \tau|^{\sigma}}
$$

is convergent and the definition (4.01) of $\Psi_{3, \sigma}(\tau)$ has meaning. Moreover by (4.01), (5.05), and the absolute convergence of (5.06) we have

$$
\Psi_{3, \sigma}(\tau)=1+\sum_{n=-\infty}^{\infty} e^{\pi i \tau n} \frac{I(\sigma, n \beta)}{2 i \beta^{1 / 2+\sigma}} \sum_{k=1}^{\infty} B_{k}(n) k^{-\sigma} .
$$

Now by the bounds of Lemmas 5.4, 5.5, 5.6, and 5.8, the outer sum here 
converges uniformly in $\sigma$. Also by Lemmas $5.4,5.5,5.6$, and 5.9,

$$
\lim _{\sigma \rightarrow 0} \frac{I(\sigma, n \beta)}{2 i \beta^{1 / 2+\sigma}} \sum_{k=1}^{\infty} B_{k}(n) k^{-\sigma}=\left\{\begin{array}{cc}
\frac{\pi^{3 / 2}}{\Gamma(3 / 2)} n^{1 / 2} \sum_{k=1}^{\infty} B_{k}(n)=\rho_{3}(n) & \text { if } n>0 \\
0 & \text { if } n \leqq 0
\end{array}\right.
$$

Hence

$$
\lim _{\sigma \rightarrow 0} \Psi_{3, \sigma}(\tau)=1+\sum_{n=1}^{\infty} \rho_{3}(n) e^{\pi i \tau n}=\Psi_{3}(\tau),
$$

so that the desired result (5.01) is proved.

6. The case $s=3$. Behavior of $\Psi_{3}(\tau)$ under the substitution $\tau^{\prime}=-1 / \tau$. It is the purpose of this section to show that

$$
\Psi_{3, \sigma}(-1 / \tau)=(-i \tau)^{3 / 2}|-i \tau|^{\sigma} \Psi_{3, \sigma}(\tau) .
$$

Once we prove this result, it will follow from the limit property (5.01) that

$$
\Psi_{3}(-1 / \tau)=(-i \tau)^{3 / 2} \Psi_{3}(\tau)
$$

We take the second form of the definition (4.01) of $\Psi_{3, \sigma}(\tau)$, namely,

$$
\Psi_{3, \sigma}(\tau)=1+\frac{1}{(-i \tau)^{3 / 2}|-i \tau|^{\sigma}}+\frac{1}{2} \sum_{k \neq 0} \sum_{h \neq 0} \frac{\eta(h, k)^{3}}{(h i-k i \tau)^{3 / 2}|h i-k i \tau|^{\sigma}},
$$

where the iterated sum has been proved convergent in the preceding section. We have

$$
\begin{aligned}
\Psi_{3, \sigma}\left(-\frac{1}{\tau}\right)= & \left(\frac{i}{\tau}\right)^{-3 / 2}\left|\frac{i}{\tau}\right|^{-\sigma}\left\{\left(\frac{i}{\tau}\right)^{3 / 2}\left|\frac{i}{\tau}\right|^{\sigma}+1\right. \\
& \left.\quad+\frac{1}{2} \sum_{k \neq 0} \sum_{h \neq 0} \frac{\eta(h, k)^{3}}{(k+h \tau)^{3 / 2}|k+h \tau|^{\sigma}}\right\} \\
= & (-i \tau)^{3 / 2}|-i \tau|^{\sigma}\left\{\frac{1}{(-i \tau)^{3 / 2}|-i \tau|^{\sigma}}+1\right. \\
& \left.\quad+\frac{1}{2} \sum_{h \neq 0} \sum_{k \neq 0} \frac{\eta(-k, h)^{3}}{(h-k \tau)^{3 / 2}|h-k \tau|^{\sigma}}\right\} .
\end{aligned}
$$

Thus by (2.05)

$$
\begin{aligned}
\Psi_{3, \sigma}\left(-\frac{1}{\tau}\right)=(-i \tau)^{3 / 2}|-i \tau|^{\sigma}\{1 & +\frac{1}{(-i \tau)^{3 / 2}|-i \tau|^{\sigma}} \\
& \left.+\frac{1}{2} \sum_{h \neq 0} \sum_{k \neq 0} \frac{\eta(h, k)^{3}}{(h i-k i \tau)^{3 / 2}|h i-k i \tau|^{\sigma}}\right\} .
\end{aligned}
$$

Hence the desired relation (6.01) will follow if we can show that the order 
of summation in the iterated sum here can be reversed.

For the remainder of this section $\sigma$ and $\tau$ will be fixed, so let us put

$$
f(h, k)=\frac{1}{(h i-k i \tau)^{3 / 2}|h i-k i \tau|^{\sigma}}, \quad g(h, k)=\eta(h, k)^{3} f(h, k) .
$$

Then to prove (6.01) it suffices to show that $\sum_{k \neq 0} \sum_{h \neq 0} g(h, k)$ $=\sum_{h \neq 0} \sum_{k \neq 0} g(h, k)$, that is,

(6.03) $\lim _{M \rightarrow \infty} \sum_{0<|k|<M} \sum_{0<|h|<\infty} g(h, k)=\lim _{M \rightarrow \infty} \sum_{0<|h|<M} \sum_{0<|k|<\infty} g(h, k)$,

where we know from $\S 5$ that the limits exist. Now we claim that (6.03) will follow if we can demonstrate

$$
\begin{aligned}
& \lim _{M \rightarrow \infty} \sum_{0<|k|<M} \sum_{M \leqq h<\infty} g(h, k)=0, \\
& \lim _{M \rightarrow \infty} \sum_{0<|h|<M} \sum_{M \leqq k<\infty} g(h, k)=0 .
\end{aligned}
$$

For if (6.04) and (6.05) hold, then, in view of the known existence of the limits in (6.03), we can assert that both sides of (6.03) are equal to $\lim _{M \rightarrow \infty} \sum_{0<|k|<M} \sum_{0<|h|<M} g(h, k)$. Thus it suffices to prove (6.04) and (6.05). Before doing this we establish some lemmas.

Lemma 6.1. If $|k|$ is not a square and if $a$ and $b$ are both positive or both negative, then

$$
\left|\sum_{a \leqq h \leqq b} \eta(h, k)^{3}\right|<48|k|^{1 / 2} \log |k| .
$$

In view of (2.02), it suffices to prove the lemma for positive $k$. Our tools are, of course, (2.01) and Lemma 2.4. If $k$ is odd we have

$$
\left|\sum_{a \leqq h \leqq b} \eta(h, k)^{3}\right|=\left|e^{-3 \pi i(k-1) / 4} \sum_{a / 2 \leqq j \leqq b / 2}\left(\frac{2 j}{k}\right)\right|<2 k^{1 / 2} \log k .
$$

For even $k$ we have

$$
\begin{aligned}
\left|\sum_{a \leqq h \leqq b} \eta(h, k)^{3}\right| & =\left|\sum_{\mu=1,3,5,7} \sum_{a \leqq \mu+8 \nu \leqq b} \eta(\mu+8 \nu, k)^{3}\right| \\
& =\left|\sum_{\mu=1,3,5,7} e^{3 \pi i \mu / 4} \sum_{(a-\mu) / 8 \leqq \nu \leqq(b-\mu) / 8}\left(\frac{k}{|\mu+8 \nu|}\right)\right| \\
& <\sum_{\mu=1,3,5,7} 2(4 k)^{1 / 2} \log (4 k) \leqq 48 k^{1 / 2} \log k,
\end{aligned}
$$

inasmuch as when $k$ is positive and not a square $(k / m)$ is (for positive $m$ ) a nonprincipal residue character modulo $4 k$. Thus Lemma 6.1 is proved. 
LEMMA 6.2. If $|h|$ is not a square and if $a$ and $b$ are both positive or both negative, then

$$
\left|\sum_{a \leqq k \leqq b} \eta(h, k)^{3}\right|<48|h|^{1 / 2} \log |h| .
$$

By (2.02) we may assume $h$ positive. By (2.03) we have

$$
\sum_{a \leqq k \leqq b} \eta(h, k)^{3}=e^{3 \pi i / 4} \sum_{a \leqq k \leqq b} \eta(-k, h)^{3} .
$$

Thus Lemma 6.2 follows from Lemma 6.1.

Throughout the remainder of this section let us put $\tau=\alpha+i \beta$, where $\alpha$ is real and $\beta$ is real and positive.

Lemma 6.3. For $0<\sigma \leqq 1 / 2$, we have

$$
|f(h, k+1)-f(h, k)|<\frac{2|\tau|}{\left(\{h-(k+\theta) \alpha\}^{2}+\{(k+\theta) \beta\}^{2}\right)^{5 / 4+\sigma / 2}},
$$

where $0 \leqq \theta \leqq 1$. (Of course $\theta$ depends upon $h, k$, and $\tau$.)

This follows from the first mean-value theorem of integral calculus:

$$
\begin{aligned}
\mid f(h, k+1) & -f(h, k) \mid \\
& \leqq \max _{0 \leqq u \leqq 1}\left|\frac{d}{d u}\{h i-(k+u) i \tau\}^{-(3+\sigma) / 2}\{-h i+(k+u) i \bar{\tau}\}^{-\sigma / 2}\right| \\
& \leqq\left(\frac{3}{2}+\sigma\right)|\tau| \max _{0 \leqq u \leqq 1}|h i-(k+u) i \tau|^{-(5 / 2+\sigma)} \\
& =\left(\frac{3}{2}+\sigma\right)|\tau||h i-(k+\theta) i \tau|^{-(5 / 2+\sigma)} .
\end{aligned}
$$

Lemma 6.4. For $0<\sigma \leqq 1 / 2$, we have

$$
|f(h+1, k)-f(h, k)|<\frac{2}{\left\{\left(h+\theta^{\prime}-\alpha k\right)^{2}+k^{2} \beta^{2}\right\}^{5 / 4+\sigma / 2}},
$$

where $\theta^{\prime}$ is a number between 0 and 1 inclusive (depending on $h, k$, and $\tau$ ).

The proof is like that of Lemma 6.3.

Now we proceed to prove (6.04). Let $M$ be a large positive integer and let $k$ be an integer such that $0<|k|<M$. If we put $\sum_{a \leqq h \leqq b} \eta(h, k)^{3}=T_{k}(a, b)$, we have by partial summation

$$
\sum_{h=M}^{\infty} g(h, k)=\sum_{h=M}^{\infty} \eta(h, k)^{3} f(h, k)=\sum_{h=M}^{\infty} T_{k}(M, h)\{f(h, k)-f(h+1, k)\} .
$$


A similar identity holds for $\sum_{h=-\infty}^{-M} g(h, k)$.

Hence if $|k|$ is not a square we have by Lemmas 6.1 and 6.4

$$
\left|\sum_{h=M}^{\infty} g(h, k)\right|<\sum_{h=M}^{\infty} \frac{96|k|^{1 / 2} \log |k|}{\left\{\left(h+\theta^{\prime}-\alpha k\right)^{2}+k^{2} \beta^{2}\right\}^{5 / 4+\sigma / 2}} .
$$

We split the sum on the right into two parts $S_{1}$ and $S_{2}$, according as

$$
M \leqq h<(|\alpha|+1) M \text { or }(|\alpha|+1) M \leqq h<\infty .
$$

In $S_{2}$ we have

$$
\begin{aligned}
h+\theta^{\prime}-\alpha k & \geqq h-|\alpha k| \\
& =h\left(1-\frac{|\alpha k|}{h}\right) \\
& \geqq h\left(1-\frac{|\alpha| M}{(|\alpha|+1) M}\right) \\
& =\frac{h}{|\alpha|+1} .
\end{aligned}
$$

Thus

$$
\begin{aligned}
S_{2} & <96|k|^{1 / 2}(\log |k|)(|\alpha|+1)^{5 / 2+\sigma} \sum_{(|\alpha|+1) M \leqq h<\infty} h^{-5 / 2-\sigma} \\
& <96|k|^{1 / 2}(\log |k|)(|\alpha|+1) M^{-3 / 2-\sigma} .
\end{aligned}
$$

We split the sum $S_{1}$ into two parts $S_{1}^{\prime}$ and $S_{1}^{\prime \prime}$ according as

$$
\left|h+\theta^{\prime}-\alpha k\right|<M /(|\alpha|+1) \text { or }\left|h+\theta^{\prime}-\alpha k\right| \geqq M /(|\alpha|+1) \text {. }
$$

If $S_{1}^{\prime}$ is nonempty, we must have $|k| \geqq M /(|\alpha|+1)$; hence

$$
\begin{aligned}
S_{1}{ }^{\prime} & <\sum_{M \leqq h<(|\alpha|+1) M} \frac{96|k|^{1 / 2} \log |k|}{\{M \beta /(|\alpha|+1)\}^{5 / 2+\sigma}} \\
& <96|k|^{1 / 2}(\log |k|)(|\alpha|+1)^{7^{7 / 2+\sigma} \beta^{-5 / 2-\sigma}} M^{-3 / 2-\sigma} .
\end{aligned}
$$

For $S_{1}^{\prime \prime}$ we have

$$
\begin{aligned}
S_{1}{ }^{\prime \prime} & <\sum_{M \leqq h<(|\alpha|+1) M} \frac{96|k|^{1 / 2} \log |k|}{\{M /(|\alpha|+1)\}^{5 / 2+\sigma}} \\
& <96|k|^{1 / 2}(\log |k|)(|\alpha|+1)^{7 / 2+\sigma} M^{-3 / 2-\sigma} .
\end{aligned}
$$

Hence if $|k|$ is not a square, we have

$$
\left|\sum_{h=M}^{\infty} g(h, k)\right|<C_{1}(\tau)|k|^{1 / 2}(\log |k|) M^{-3 / 2-\sigma} \text {. }
$$


Similarly if $|k|$ is not a square

$$
\left|\sum_{h=-\infty}^{-M} g(h, k)\right|<C_{1}(\tau)|k|^{1 / 2}(\log |k|) M^{-3 / 2-\sigma} .
$$

If $|k|$ is a square, the estimate $\left|T_{k}(a, b)\right|<48|k|^{1 / 2} \log |k|$ must be replaced by some other argument. Instead of using partial summation we merely estimate directly:

$$
\left|\sum_{h=M}^{\infty} g(h, k)\right|<\sum_{h=M}^{\infty} \frac{1}{\left\{(h-k \alpha)^{2}+k^{2} \beta^{2}\right\}^{3 / 4+\sigma / 2}} .
$$

By breaking the sum on the right into parts exactly as above we get

$$
\left|\sum_{h=M}^{\infty} g(h, k)\right|<C_{2}(\tau) M^{-1 / 2-\sigma} .
$$

Similarly

$$
\left|\sum_{h=-\infty}^{-M} g(h, k)\right|<C_{2}(\tau) M^{-1 / 2-\sigma} .
$$

Now there are less than $2 M^{1 / 2}$ integers $k$ such that $0<|k|<M$ and $|k|$ is a square. Hence

$$
\begin{aligned}
&\left|\sum_{0<|k|<M}\left\{\sum_{h=M}^{\infty} g(h, k)+\sum_{h=-\infty}^{-M} g(h, k)\right\}\right| \\
& \quad<\sum_{0<|k|<M} 2 C_{1}(\tau)|k|^{1 / 2}(\log |k|) M^{-3 / 2-\sigma}+4 M^{1 / 2} C_{2}(\tau) M^{-1 / 2-\sigma} \\
&<\left\{4 C_{1}(\tau) \log M+4 C_{2}(\tau)\right\} M^{-\sigma} .
\end{aligned}
$$

But the last expression tends to zero with increasing $M$. Thus (6.04) is proved.

We turn to proving (6.05). Let $M$ be a large positive integer and let $h$ be an integer such that $0<|h|<M$. If $|h|$ is not a square, we find by partial summation as above (using Lemmas 6.2 and 6.3 instead of Lemmas 6.1 and 6.4)

$$
\begin{aligned}
\left|\sum_{k=M}^{\infty} g(h, k)\right| & <\sum_{k=M}^{\infty} \frac{96|\tau||h|^{1 / 2} \log |h|}{\left(\{h-(k+\theta) \alpha\}^{2}+\{(k+\theta) \beta\}^{2}\right)^{5 / 4+\sigma / 2}} \\
& <96|\tau||h|^{1 / 2}(\log |h|) \beta^{-5 / 2-\sigma} \sum_{k=M}^{\infty} k^{-5 / 2-\sigma} \\
& <C_{3}(\tau)|h|^{1 / 2}(\log |h|) M^{-3 / 2-\sigma} .
\end{aligned}
$$

If $|h|$ is a square, we have the direct estimate 


$$
\begin{aligned}
\left|\sum_{k=M}^{\infty} g(h, k)\right| & <\sum_{k=M}^{\infty}\left\{(h-k \alpha)^{2}+k^{2} \beta^{2}\right\}^{-3 / 4-\sigma / 2}<\sum_{k=M}^{\infty}(k \beta)^{-3 / 2-\sigma} \\
& <C_{4}(\tau) M^{-1 / 2-\sigma} .
\end{aligned}
$$

Since $\sum_{k=-\infty}^{-M} g(h, k)$ clearly has the same estimates, we have

$$
\begin{aligned}
&\left|\sum_{0<|h|<M}\left\{\sum_{k=M}^{\infty} g(h, k)+\sum_{k=-\infty}^{-M} g(h, k)\right\}\right| \\
&<\sum_{0<|h|<M} 2 C_{3}(\tau)|h|^{1 / 2}(\log |h|) M^{-3 / 2-\sigma}+4 M^{1 / 2} C_{4}(\tau) M^{-1 / 2-\sigma} \\
&<\left\{4 C_{3}(\tau) \log M+4 C_{4}(\tau)\right\} M^{-\sigma} .
\end{aligned}
$$

Since the last expression tends to zero as $M \rightarrow \infty,(6.05)$ is proved.

Thus all the numbered statements in this section are proved.

7. The case $s=3$. Behavior of $\Psi_{3}(1-1 / \tau)$ as $\tau \rightarrow i \infty$. In this section we study $\Psi_{3, \sigma}(1-1 / \tau)$ in order to determine the behavior of $\Psi_{3}(1-1 / \tau)$ as $\tau \rightarrow i \infty$. We begin with a lemma which will enable us to perform a reversal of order of summation.

LEMMA 7.1. If $h$ is an odd positive integer not a square, then

$$
\left|\sum_{a \leqq k \leqq b}\left(\frac{k}{h}\right) e^{-3 \pi i h(k-1) / 4}\right|<16 h^{1 / 2} \log h .
$$

If $a>0, b>0$, and $k$ is an integer which is not a square, then

$$
\left|\sum_{a \leqq h \leqq b, h \text { odd }}\left(\frac{k}{h}\right) e^{-3 \pi i h(k-1) / 4}\right|<16|k|^{1 / 2} \log |4 k| .
$$

This lemma is easily deduced from Lemma 2.4. (Cf. the second half of the proof of Lemma 6.1.)

Now we proceed. By (4.01) and Lemma 2.2 we have

$$
\begin{aligned}
\Psi_{3, \sigma}(1+\tau) & =1+\sum_{k=1}^{\infty} \sum_{h=-\infty}^{\infty} \frac{\eta(h+k, k)^{3}}{(h i-k i \tau)^{3 / 2}|h i-k i \tau|^{\sigma}} \\
& =1+\sum_{k=1}^{\infty} \sum_{h \text { odd }}\left(\frac{k}{|h|}\right)^{3} \frac{e^{-3 \pi i h(k-1) / 4}}{(h i-k i \tau)^{3 / 2}|h i-k i \tau|^{\sigma}}
\end{aligned}
$$

Thus by Lemma 2.3,

$$
\Psi_{3, \sigma}(1+\tau)=\sum_{k=-\infty}^{\infty} \sum_{h \text { odd }>0}\left(\frac{k}{h}\right)^{3} \frac{e^{-3 \pi i h(k-1) / 4}}{(h i-k i \tau)^{3 / 2}|h i-k i \tau|^{\sigma}}
$$

Now by using Lemma 7.1 and proceeding exactly as in $\S 6$ it is possible to prove that the order of summation here can be reversed. Thus 


$$
\begin{aligned}
\Psi_{3, \sigma}\left(1-\frac{1}{\tau}\right)= & (-i \tau)^{3 / 2}|-i \tau| \sigma \sum_{h \text { odd }>0} \sum_{k}\left(\frac{k}{h}\right)^{3} \frac{e^{-3 \pi i h(k-1) / 4}}{(h \tau+k)^{3 / 2}|h \tau+k|^{\sigma}} \\
= & (-i \tau)^{3 / 2}|-i \tau|_{h \text { odd }>0}^{\sigma} \sum_{k}\left(\frac{k}{h}\right)^{3} \frac{e^{-3 \pi i(h k-h+1) / 4}}{(-h i \tau-k i)^{3 / 2}|-h i \tau-k i|^{\sigma}} \\
= & (-i \tau)^{3 / 2}|-i \tau|_{h \text { odd }>0}^{\sigma} e^{3 \pi i(h-1) / 4} \sum_{k \bmod 8 h}\left(\frac{k}{h}\right)^{3} e^{-3 \pi i h k / 4} \\
& \cdot \sum_{m}\{-h i \tau-(k-8 h m) i\}^{-3 / 2}|-h i \tau-(k-8 h m) i|^{-\sigma} .
\end{aligned}
$$

By applying Lemma 5.7 we get

$$
\begin{aligned}
\Psi_{3, \sigma}\left(1-\frac{1}{\tau}\right)= & (-i \tau)^{3 / 2}|-i \tau| \sigma \sum_{h \text { odd }>0} e^{3 \pi i(h-1) / 4} \sum_{k \bmod 8 h}\left(\frac{k}{h}\right)^{3} e^{-3 \pi i h k / 4} \\
& \cdot(4 h)^{-3 / 2-\sigma} \sum_{m} \frac{I(\sigma, m \beta / 4)}{2 i(\beta / 4)^{1 / 2+\sigma}} e^{\pi i \mid \tau / 4+k /(4 h)\} m} .
\end{aligned}
$$

In view of (2.14) this becomes

$$
\begin{aligned}
\Psi_{3, \sigma}\left(1-\frac{1}{\tau}\right) & =(-i \tau)^{3 / 2}|-i \tau|^{\sigma} \sum_{h \text { odd }>0} \sum_{m} e^{2 \pi i \tau m / 8} \frac{I(\sigma, m \beta / 4)}{i \beta^{1 / 2+\sigma}} D_{h}(m) h^{-\sigma} \\
& =(-i \tau)^{3 / 2}|-i \tau|^{\sigma} \sum_{h \text { odd }>0} \sum_{m \equiv 3(\bmod 8)} e^{2 \pi i \tau m / 8} \frac{I(\sigma, m \beta / 4)}{i \beta^{1 / 2+\sigma}} B_{h}(m) h^{-\sigma} .
\end{aligned}
$$

Now by proceeding exactly as in $\$ 5$ it is possible to show that we may permute the summations on $h$ and $m$ and then go to the limit term by term. (Actually the process is simpler here, for, since $m \equiv 3(\bmod 8),-m$ cannot be a square, and thus one of the troublesome points of $\$ 5$ does not arise here.) Hence we have (by Lemma 5.9)

$$
\begin{aligned}
& \Psi_{3}\left(1-\frac{1}{\tau}\right) \\
& \quad=(-i \tau)^{3 / 2} \sum_{m>0, m \equiv 3(\bmod 8)} e^{2 \pi i \tau m / 8} \frac{(2 \pi)^{3 / 2}}{\Gamma(3 / 2)}\left(\frac{1}{8} m\right)^{1 / 2} \sum_{h \text { odd }>0} B_{h}(m) \\
& \quad=(-i \tau)^{3 / 2} e^{3 \pi i \tau / 4} \sum_{n=0}^{\infty} e^{2 \pi i \tau n} \frac{(2 \pi)^{3 / 2}}{\Gamma(3 / 2)}\left(n+\frac{3}{8}\right)^{1 / 2} \sum_{h \text { odd }>0} B_{h}(8 n+3) .
\end{aligned}
$$

In view of (6.02) and (7.01) we may now conclude by the method sketched in $\S 2$ that $\vartheta_{3}(0 \mid \tau)^{3}$ and $\Psi_{3}(\tau)$ are identical. Thus

$$
r_{3}(n)=\rho_{3}(n)
$$

$$
(n=1,2, \cdots) \text {, }
$$

where $\rho_{3}(n)$ is defined by (1.01) and has the evaluation (4.08). Now clearly 


$$
r_{3}(n)=\sum_{d^{2} \mid n} R_{3}\left(n / d^{2}\right)
$$

where $R_{3}(n)$ is the number of primitive representations of $n$ as the sum of three squares (that is, representations in which the only common divisor of the three squares is unity). Thus (7.02), (7.03), and (4.10) imply that

$$
R_{3}(n)=\rho_{3}(n)
$$$$
(n=1,2, \cdots) \text {, }
$$

where $\rho_{3}(n)$ is given by (4.09). We combine (7.02) and (7.04) in the following theorem, the main result of this paper.

THEOREM B. For any positive integer $n$ we have

$$
\begin{aligned}
r_{3}(n)=\rho_{3}(n)=\frac{16}{\pi} n^{1 / 2} \chi_{2}(n) K(-4 n) & \prod_{p^{2} \mid n}\left(1+\frac{1}{p}+\cdots\right. \\
& \left.+\frac{1}{p^{b-1}}+\frac{1}{p^{b}}\left\{1-\left(\frac{-p^{-2 b} n}{p}\right) \frac{1}{p}\right\}^{-1}\right),
\end{aligned}
$$

where $b=b(p)$ is the largest integer such that $p^{2 b} \mid n$,

$$
K(-4 n)=\sum_{n=1}^{\infty}\left(\frac{-4 n}{m}\right) \frac{1}{m},
$$

and, if $4^{a}$ is the highest power of 4 dividing $n$,

$$
\chi_{2}(n)= \begin{cases}0 & \text { if } 4^{-a} n \equiv 7(\bmod 8) \\ 1 / 2^{a} & \text { if } 4^{-a} n \equiv 3(\bmod 8) \\ 3 / 2^{a+1} & \text { if } 4^{-a} n \equiv 1,2,5,6(\bmod 8)\end{cases}
$$

Further,

$$
R_{3}(n)=P_{3}(n)=\frac{G_{n}}{\pi} n^{1 / 2} K(-4 n)
$$

where

$$
G_{n}=\left\{\begin{array}{cl}
0 & \text { if } n \equiv 0,4,7(\bmod 8), \\
16 & \text { if } n \equiv 3(\bmod 8), \\
24 & \text { if } n \equiv 1,2,5,6(\bmod 8) .
\end{array}\right.
$$

Since $\vartheta_{3}(0 \mid 1-1 / \tau)^{3}$ and $\Psi_{3}(1-1 / \tau)$ are identical, we have by $(2.15)$ and (7.01)

$$
\left(\sum_{n=-\infty}^{\infty} e^{2 \pi i r n(n+1) / 2}\right)^{3}=\sum_{n=0}^{\infty} e^{2 \pi i \tau n} \frac{(2 \pi)^{3 / 2}}{\Gamma(3 / 2)}\left(n+\frac{3}{8}\right)^{1 / 2} \sum_{n \text { odd }>0} B_{h}(8 n+3) .
$$

In other words, the number of representations of a non-negative integer $n$ 
as the sum of three triangular numbers is given by the positive value (7.05) $\frac{(2 \pi)^{3 / 2}}{\Gamma(3 / 2)}\left(n+\frac{3}{8}\right)^{1 / 2} \sum_{h \text { odd }>0} B_{h}(8 n+3)=2 \pi(8 n+3)^{1 / 2} \sum_{h=1}^{\infty} B_{h}(8 n+3)$.

(We have used the fact that $\chi_{2}(8 n+3)=1$.) This side result is not surprising, since it is easy to see directly that the number of representations of $n$ as the sum of three triangular numbers is equal to $r_{3}(8 n+3)$, which, by the theorem just proved, is equal to the right-hand expression in (7.05).

\section{BIBLIOGRAPHY}

\section{Paul BachmanN}

1. Die analytische Zahlentheorie, Leipzig, 1894.

2. Die Arithmetik von quadratischen Formen, part I, Leipzig, 1898.

P. T. BATEMAN

1. On the representations of a number as the sum of three squares, Bull. Amer. Math. Soc. Abstract 52-9-262.

S. CHOWLA

1. On the k-analogue of a result in the theory of the Riemann zeta function, Math. Zeit. vol. 38 (1934) pp. 483-487.

L. E. Dickson

1. History of the theory of numbers, vol. 2, Washington, 1920.

2. Studies in the theory of numbers, Chicago, 1930.

T. EstermanN

1. On the representations of a number as the sum of three or more products, Proc. London Math. Soc. (2) vol. 34 (1932) pp. 190-195.

2. On the representations of a number as a sum of squares, Acta Arithmetica vol. 2 (1936) pp. 47-79.

L. R. FORD

1. Automorphic functions, New York, 1929.

G. H. HARDY

1. On the representation of a number as the sum of any number of squares, and in particular of five or seven, Proc. London Math. Soc. (2) vol. 17 (1918) pp. xxii-xxiv (Records of proceedings at the meeting of March 14, 1918).

2. On the representation of a number as the sum of any number of squares, and in particular of five or seven, Proc. Nat. Acad. Sci. U.S.A. vol. 4 (1918) pp. 189-193.

3. On the representation of a number as the sum of any number of squares, and in particular of five, Trans. Amer. Math. Soc. vol. 21 (1920) pp. 255-284. For a list of errata see vol. 29 (1927) pp. 845-847.

4. Note on Ramanujan's trigonometrical function $C_{g}(n)$, and certain series of arithmetical functions, Proc. Cambridge Philos. Soc. vol. 20 (1921) pp. 263-271.

5. Ramanujan, twelve lectures on subjects suggested by his life and work, Cambridge, 1940.

G. H. HARDY AND E. M. WRIGHT

1. An introduction to the theory of numbers, Oxford, 1938, 1945.

\section{ERICH HeCKE}

1. Darstellung von Klassenzahlen als Perioden von Integralen 3. Gattung aus dem Gebiet der elliptischen Modulfunktionen, Abh. Math. Sem. Hamburgischen Univ. vol. 4 (1925) pp. 211-223.

2. Theorie der Eisensteinschen Reihen höherer Stufe und ihre Anwendung auf Funktionen- 
theorie und Arithmetik, Abh. Math. Sem. Hamburgischen Univ. vol. 5 (1927) pp. 199224.

MARK KAC

1. Almost periodicity and the representation of integers as sums of squares, Amer. J. Math. vol. 62 (1940) pp. 122-126.

H. D. KLoostermann

1. On the representations of numbers in the form $a x^{2}+b y^{2}+c z^{2}+d t^{2}$, Proc. London Math. Soc. (2) vol. 25 (1926) pp. 143-173.

2. On the representations of numbers in the form $a x^{2}+b y^{2}+c z^{2}+d t^{2}$, Acta Math. vol. 49 (1927) pp. 407-464.

EDMUND LANDAU

1. Über die Einteilung der positiven ganzen Zahlen in vier Klassen nach der Mindestzahl der $z u$ ihrer additiven Zusammensetzung erforderlichen Quadrate, Archiv der Mathematik und Physik (3) vol. 13 (1908) pp. 305-312.

2. Handbuch der Lehre von der Verteilung der Primzahlen, Leipzig, 1909.

3. Abschätzungen von Charaktersummen, Einheiten und Klassenzahlen, Nachr. Ges. Wiss. Göttingen (1918) pp. 79-97.

4. Vorlesungen über Zahlentheorie, vol. 1, Leipzig, 1927.

GEORG LANDSBERG

1. Zur Theorie der Gausschen Summen und des linearen Transformationen der Thetafunktionen, J. Reine Angew. Math. vol. 111 (1893) pp. 234-253.

R. LiPSCHITZ

1. Untersuchung einer aus vier Elementen gebildeten Reihe, J. Reine Angew. Math. vol. 54 (1857) pp. 313-328.

L. J. MORDELL

1. On the representation of numbers as a sum of $2 r$ squares, Quarterly Journal of Pure and Applied Mathematics vol. 48 (1917) pp. 93-104.

2. On the representations of a number as a sum of an odd number of squares, Transactions of the Cambridge Philosophical Society vol. 22 (1919) pp. 361-372.

3. Poisson's summation formula and the Riemann zeta function, J. London Math. Soc. vol. 4 (1929) pp. 285-291.

G. Pólya

1. Über die Verteilung der quadratischen Reste und Nichtreste, Nachr. Ges. Wiss. Göttingen (1918) pp. 21-29.

S. Ramanujan

1. On certain trigonometrical sums and their applications in the theory of numbers, Transactions of the Cambridge Philosophical Society vol. 22 (1918) pp. 259-276.

J. SCHUR

1. Einige Bemerkungen zu der vorstehenden Arbeit des Herrn G. Pólya, Nachr. Ges. Wiss. Göttingen (1918) pp. 30-36.

C. L. SIEgEL

1. Über die analytischen Theorie der quadratischen Formen, Ann. of Math. (2) vol. 36 (1935) pp. 527-606.

G. K. Stanley

1. On the representations of a number as the sum of seven squares, J. London Math. Soc. vol. 2 (1927) pp. 91-96.

E. T. Whittaker and E. M. Watson

1. A course of modern analysis, Cambridge, 1927.

University of Pennsylvania

Philadel phia, Pa. 